\title{
Whole genome sequencing analysis identifies recurrent structural alterations in esophageal squamous cell carcinoma
}

\author{
Munmee Dutta ${ }^{1,2}$, Hidewaki Nakagawa ${ }^{\text {Corresp., } 3}{ }^{3}$,Hiroaki Kato ${ }^{4}$, Kazuhiro Maejima ${ }^{3}$, Shota Sasagawa ${ }^{3}$, Kaoru \\ Nakano $^{3}$, Aya Sasaki-Oku ${ }^{3}$, Akihiro Fujimoto ${ }^{5}$, Raúl Nicolás Mateos ${ }^{1,2}{ }^{2}$, Ashwini Patil ${ }^{2}$, Hiroko Tanaka ${ }^{2}$, Satoru \\ Miyano $^{2,6}$, Takushi Yasuda ${ }^{4}$, Kenta Nakai ${ }^{1,2}$, Masashi Fujita ${ }^{\text {Corresp. } 3}$ \\ ${ }^{1}$ Department of Computational Biology and Medical Sciences, Graduate school of Frontier Sciences, The University of Tokyo, Chiba, Japan \\ 2 Human Genome Center, The Institute of Medical Science, The University of Tokyo, Tokyo, Japan \\ 3 Laboratory for Cancer Genomics, RIKEN Center for Integrative Medical Sciences, Yokohama, Japan \\ 4 Department of Surgery, Faculty of Medicine, Kindai University, Osaka, Japan \\ 5 Department of Drug Discovery Medicine, Kyoto University Graduate School of Medicine, Kyoto, Japan \\ 6 Health Intelligence Center, The Institute of Medical Science, The University of Tokyo, Tokyo, Japan \\ Corresponding Authors: Hidewaki Nakagawa, Masashi Fujita \\ Email address: hidewaki@riken.jp, m-fujita@riken.jp
}

Esophageal squamous cell carcinoma (ESCC) is the predominant type of esophageal cancer in the Asian region, including Japan. A previous study reported mutational landscape of Japanese ESCCs by using exome sequencing. However, somatic structural alterations were yet to be explored. To provide a comprehensive mutational landscape, we performed whole genome sequencing (WGS) analysis of biopsy specimens from 20 ESCC patients in a Japanese population. WGS analysis identified non-silent coding mutations of TP53, ZNF750 and FAT1 in ESCC. We detected six mutational signatures in ESCC, one of which showed significant association with smoking status. Recurrent structural variations, many of which were chromosomal deletions, affected genes such as $\angle R P 1 B, T T C 28$, CSMD1, PDE4D, SDK1 and WWOX in $25 \%-30 \%$ of tumors. Somatic copy number amplifications at 11q13.3 (CCND1), 3q26.33 (TP63/SOX2), and 8p11.23 (FGFR1) and deletions at 9p21.3 (CDKN2A) were identified. Overall, these multi-dimensional view of genomic alterations improve the understanding of the ESCC development at molecular level and provides future prognosis and therapeutic implications for ESCC in Japan. 


\section{Whole genome sequencing analysis identifies}

2 recurrent structural alterations in esophageal

3 squamous cell carcinoma

4

5

6 8

9

Munmee Dutta ${ }^{1,2}$, Hidewaki Nakagawa ${ }^{3}$, Hiroaki Kato ${ }^{4}$, Kazuhiro Maejima $^{3}$, Shota Sasagawa ${ }^{3}$, Kaoru Nakano ${ }^{3}$, Aya Sasaki-Oku³, Akihiro Fujimoto ${ }^{5}$, Raúl Nicolás Mateos ${ }^{1,2}$, Ashwini Patil², Hiroko Tanaka ${ }^{2}$, Satoru Miyano ${ }^{2,6}$, Takushi Yasuda ${ }^{4}$, Kenta Nakai $^{1,2}$, and Masashi Fujita ${ }^{3}$

${ }^{1}$ Department of Computational Biology and Medical Sciences, Graduate school of Frontier Sciences, The University of Tokyo, 5-1-5 Kashiwanoha, Kashiwa-shi, Chiba 277-8561 Japan ${ }^{2}$ Human Genome Center, The Institute of Medical Science, The University of Tokyo, 4-6-1 Shirokanedai, Minato-ku, Tokyo 108-0069, Japan

${ }^{3}$ Laboratory for Cancer Genomics, RIKEN Center for Integrative Medical Sciences, Yokohama, Japan

${ }^{4}$ Department of Surgery, Faculty of Medicine, Kindai University School, Osaka, Japan

${ }^{5}$ Department of Drug Discovery Medicine, Kyoto University Graduate School of Medicine, Kyoto, Japan

${ }^{6}$ Health Intelligence Center, The Institute of Medical Science, The University of Tokyo, Tokyo, Japan

Corresponding Author:

Masashi Fujita, PhD and Hidewaki Nakagawa, MD, PhD

Laboratory for Cancer Genomics,

RIKEN Center for Integrative Medical Sciences,

1-7-22 Suehiro-cho, Tsurumi-ku, Yokohama, Kanagawa, 230-0045, Japan

Tel: $+81-45-503-9288$

FAX: +81-45-503-9294

Email address: m-fujita@riken.jp, and hidewaki@riken.jp 
33

34 35

\section{Abstract}

Esophageal squamous cell carcinoma (ESCC) is the predominant type of esophageal cancer in the Asian region, including Japan. A previous study reported mutational landscape of Japanese ESCCs by using exome sequencing. However, somatic structural alterations were yet to be explored. To provide a comprehensive mutational landscape, we performed whole genome sequencing (WGS) analysis of biopsy specimens from 20 ESCC patients in a Japanese population. WGS analysis identified non-silent coding mutations of TP53, ZNF750 and FAT1 in ESCC. We detected six mutational signatures in ESCC, one of which showed significant association with smoking status. Recurrent structural variations, many of which were chromosomal deletions, affected genes such as LRP1B, TTC28, CSMD1, PDE4D, SDK1 and $W W O X$ in $25 \%-30 \%$ of tumors. Somatic copy number amplifications at $11 q 13.3$ (CCND1), $3 q 26.33$ (TP63/SOX2), and 8p11.23 (FGFR1) and deletions at 9p21.3 (CDKN2A) were identified. Overall, these multi-dimensional view of genomic alterations improve the understanding of the ESCC development at molecular level and provides future prognosis and therapeutic implications for ESCC in Japan.

\section{Introduction}

1 Esophageal cancer is the eighth most aggressive cancer type and sixth most common cause of cancer-related death worldwide(Zhang et al., 2015). Esophageal cancer has two major subtypes: esophageal squamous cell carcinoma (ESCC) and esophageal adenocarcinoma (EAC).

4 The incidence rate of ESCC is high in the Asian regions including Japan, China and

5 India(Sawada et al., 2016)'(Chattopadhyay et al., 2010). On the other hand, EAC predominates in - the Western countries. Alcohol drinking and tobacco smoking are the two main risk factors of 
57 ESCC development(Zhang et al., 2015). Additionally, micronutrient deficiency and genetic

58 variants that harm the activity of alcohol-metabolizing enzymes also promote $\mathrm{ESCC}$ (Chang et 59 al., 2017). Despite the advancement of the diagnostic techniques and treatment of ESCC, the 60 survival rate is still poor.

In Japan, ESCC is most conventionally treated by the standard neo-adjuvant chemotherapy

62 followed by surgical resection. Preoperative chemotherapy with cisplatin and fluorouracil is 63 considered as standard treatment option for patients in advanced stage of ESCC(Baba et al., 64 2014)(Yoshida et al., 2018). Response rate to this standard therapy is moderate (35$6540 \%$ )(Yoshida et al., 2018). The varying response among patients might be partly attributed to 66 the genetic heterogeneity of tumors.

67 Although ESCC is common in China and Japan, ESCC of both the countries have some 68 common as well as different characteristics. Smoking and alcohol drinking are attributed as 69 concerning risk factors for ESCC of both the countries. In Japan, ESCC is the tenth most 70 common cancer type while it is the fourth most frequent cancer type in China(Sawada et al., 71 2016). ESCC incidence and mortality rates are higher in China than in Japan(Yingsong Lin, 72 Yukari Totsuka, Yutong He, Shogo Kikuchi, Youlin Qiao, Junko Ueda, Wenqiang Wei, Manami 73 Inoue, 2013). The incidence of esophageal cancer is higher in males than in females, for 74 example, 16,241 male cases and 3,778 female cases were found in Japan in 2018 (source of data: 75 World Health Organization [WHO] Global Cancer Observatory database)(Yingsong Lin, Yukari 76 Totsuka, Yutong He, Shogo Kikuchi, Youlin Qiao, Junko Ueda, Wenqiang Wei, Manami Inoue, 77 2013).

78 Recently, several studies in China and Japan characterized somatic mutations in ESCC using 79 whole-exome sequencing (WES). These WES studies reported frequent mutations of TP53, 
80

81

82

CDKN2A, NOTCH1, RB1, ERBB2 and NFE2L2 in ESCC(Sawada et al., 2016),(Song et al., 2014),(Qin et al., 2016). While somatic mutations are important, structural variations (SVs) and somatic copy number alterations (CNAs) would also affect the development of ESCC. SVs have the capability to rearrange the large genomic alterations which impact in the treatment and prediction of its consequences in patients. However, systematic characterization of somatic mutations, mutational signatures, SVs and CNAs together have not been reported in Japanese ESCC at the whole-genome level.

In this study, we investigated 20 ESCC samples in a Japanese population using WGS. We comprehensively analyzed and showed somatic mutations in important genes, mutational signatures and their association with clinical features. Our SV and CNA analysis also identified potential target genes and regions in ESCC. The characterization of the mutational landscape in Japanese ESCC will guide our understanding of the disease in a better way and provide potential targets for the precision treatment and therapeutic prevention.

\section{Materials \& Methods}

\section{Clinical samples}

Tumor and normal samples were obtained from 20 patients in Kindai University hospital, Osaka, Japan. All the patients agreed to participate in the study and provided written informed consent following ICGC guidelines. The study was approved by the Institutional Review Board at Kindai University Hospital and RIKEN (approval number 25-031) and. Personal history of ESCC in these patients were unavailable. All except one patients (OK047) had not received any cancer treatment before the sample collection. OK047 received cisplatin-based chemotherapy before sample collection. Tumor tissues in esophagus were collected by biopsy, and histologically 
103 confirmed as ESCC. The patients were treated with neoadjuvant chemotherapy after collecting

104 the samples. The clinico-pathological data are available in the Table 1 and Table S1.

105

106 Whole genome sequencing

107 We performed WGS of the 20 pairs of matched tumor and normal samples. The tumor DNA was

108 extracted from the ESCC samples, and normal DNA was from the lymphocytes in blood. The

109 libraries were prepared using TruSeq Nano DNA Library Prep Kit (Illumina) following the

110 manufacturer's protocol. Paired-end sequencing of 101- or 126-bp reads was performed using

111 HiSeq2000/2500. The Supplementary Fig. S1 shows the schematic representation of the WGS

112 analysis pipeline performed in this study. Sequence reads were mapped to the human reference

113 genome GRCh37 using BWA. We removed PCR duplicates using Picard tool

114 (http://broadinstitute.github.io/picard/).

116 Somatic mutation calling and mutation signature profiling

117 Somatic single nucleotide variations (SNVs) and short insertions/deletions (INDELs) were called

118 as previously described(Fujimoto et al., 2016). Functional annotation of the detected SNVs and

119 INDELs was performed with Annovar(Wang, Li \& Hakonarson, 2010). We applied dNdScv

120 method(Martincorena et al., 2017) to search for genes with significant recurrent mutations ( $q$ -

121 value $<0.05$ ). We further summarized and visualized the annotated variants using the MAFtools

122 package(Mayakonda et al., 2018) of the R software (https://www.r-project.org/). For the detection

123 of mutational signatures in 20 ESCC, we used the SignatureAnalyzer

124 (https://software.broadinstitute.org/cancer/cga/msp). Identified mutational signatures were 
125 compared with the COSMIC mutational signatures (version 2) using the cosine similarity

126 scoring.

127

128 Structural variation calling

129 Somatic SVs were called by merging calls of two software: in-house pipeline(Fujimoto et al., 130 2016) and Genomon2 structural variation (SV) detection tool (https://github.com/Genomon-

131 Project/GenomonSV). In the Genomon2 SV detection, The SVs were called using minimum

132 junction number 2, maximum control variant read pair 10 and minimum overhang size 50 . The

133 SVs were then filtered using parameters minimum allele frequency 0.07 , maximum control

134 variant read pair 1, control depth threshold 10 and minimum overhang size 100 . The inversion

135 size threshold set to 1000 and the simple repeats were removed. Here, SVs were categorized into

136 four classes based on the mapping information for a read pair. The four classes are

137 intrachromosomal deletion, inversion and tandem duplication, and interchromosomal

138 translocation, respectively.

139 The breakage-fusion-bridge (BFB) events were identified based on the information of

140 fold-back inversion and loss of telomere(Hermetz et al., 2014). We implement the following

141 criteria in order to infer BFB: i) Inversion is single inversion (either forward or reverse) i.e.

142 without any reciprocal partner, ii) Inversions must have copy number change versus the adjacent

143 position, and iii) The two ends of the fold-back inversion must be separated by $<20 \mathrm{~kb}$. To detect

144 kataegis, we used Maftools(Mayakonda et al., 2018) package, which is defined by mutation

145 clusters of six or more consecutive mutations localized in a small region with an average inter-

146 variant distance of less than or equal to $1 \mathrm{~kb}$. Chromothripsis was inferred in ESCC samples

147 based on the criteria provided by a previous study(Korbel \& Campbell, 2013). Chromothripsis 
148 was identified in samples which show clustering of SV breakpoints, usually more than 10

149 breakpoints within $50 \mathrm{~kb}$, with regular oscillation of copy number states.

150

151 Copy number alteration calling

152 Somatic CNAs were called by analyzing read depth of matched tumor and normal using the

153 Varscan2 software(Koboldt et al., 2012). The thresholds used to call the CNAs using

154 CopyNumber function were p-value 0.001 , minimum segment size 100 and maximum segment

155 size 1000. The output of the above step was filtered using the copyCaller function. The raw

156 CNAs were segmented by the circular binary segmentation method implemented in the $\mathrm{R}$

157 package DNAcopy. The GISTIC2.0 algorithm was used to identify the significant recurrent copy

158 number amplified and deleted regions(Mermel et al., 2011).

159

160 Identification of druggable genes

161 To identify the druggable genes across the ESCC samples, we used online gene-drug interaction

162 database (DGIdb)(Griffith et al., 2013). We used the genetically altered genes as target data in

163 order to determine the druggability of the genes. The target genes were detected by different

164 method such as SNV/INDEL analysis, SV analysis and CNA analysis in this study. The database

165 provides two options to examine gene-drug interactions either by gene or by drug names. In this

166 case, we identified gene-drug interactions by providing the gene names. The genes that have at

167 least one interaction with drug target was considered as druggable gene.

168

169 Results

170 Whole genome sequencing of ESCC samples 
171 To identify the mutational events and driver genes that contributing to the development of

172 ESCC in Japanese population, we performed WGS of 20 pairs of tumor and matched blood

173 samples. The samples were collected by biopsy from the individuals before neo-adjuvant

174 chemotherapy (except OK047). Among the 20 samples, 10 samples responded well to the

175 therapy while rest 10 samples showed poor response. The average genome coverage was $43.4 \times$

176 for the tumor samples and $34.3 \times$ for blood samples, after removal of polymerase chain reaction

177 (PCR) duplicates. The WGS data was computationally analyzed to call somatic alterations of the

178 following types: single nucleotide variations (SNVs), small insertions and deletions (INDELs),

179 structural variations (SVs), and copy number alterations (CNAs). In all, WGS analysis identified

180104,534 somatic SNVs, 10,523 somatic INDELs, and 2,641 somatic SVs in the 20 tumors (Table

181 S2). In addition, WGS analysis detected 10 significant copy number altered regions in ESCC.

182

183 Recurrent coding mutations in ESCC

184 We investigated the somatic SNVs, short INDELs in the protein-coding regions and their

185 splice sites in the 20 ESCCs. Our WGS analysis identified recurrently mutated genes, including

186 previously known esophageal cancer associated oncogenes and tumor-suppressor genes. We

187 evaluated the somatic alterations by dNdSCV(Martincorena et al., 2017) method in order to find

188 out the significantly recurring mutations across the ESCC genomes. We identified significant

189 mutations in TP53 and ZNF750 genes in ESCC consistent with findings by previous studies

190 (Fig. 1 and Fig. S2). TP53 mutations were most frequent and found in 55\% of the samples

191 followed by ZNF750 (15\%). In addition, other genes that recurrently mutated in ESCC include

192 FAT1 (10\%), PTCH1 (10\%), EP300 (5\%), FAT2 (5\%), FBXW7 (5\%), KMT2D (5\%), NFE2L2

193 (5\%), NOTCH1 (5\%), PIK3CA (5\%), RB1 (5\%), RIPK4 (5\%) and TP63 (5\%). These genes were 
194 previously reported in ESCC by different studies(Zhang et al., 2015),(Sawada et al.,

195 2016),(Chang et al., 2017),(Qin et al., 2016),(Li et al., 2018),(TCGA, 2017).

196

197 The mutational signatures of ESCC

198 In order to understand mutational mechanisms of ESCC in Japan, we analyzed the mutational 199 signatures of the 20 ESCC tumors. A Bayesian variant of the non-negative matrix-factorization 200 method was applied to trinucleotide substitution patterns and extracted six mutational signatures 201 (Fig. $2 \boldsymbol{A}-\boldsymbol{F}$ ). The mutational signatures in ESCC were then compared with the signatures of the 202 Catalogue of Somatic Mutations in Cancer (COSMIC) database(Alexandrov et al., 2013) (Table 203 S3).

204 The identified Signature W1 was highly similar to the COSMIC signature 18 (cosine 205 similarity 0.933), but the biological aetiology of this signature is not known. Signature W2 was 206 characterized by $\mathrm{C}>\mathrm{A}$ and $\mathrm{C}>\mathrm{T}$ mutations and found in almost $85 \%$ of the ESCC patients (Fig. $2072 G$ ). Signature W2 was similar to the COSMIC signature 1 (cosine similarity 0.850 ), which is an 208 age-dependent signature. Signature W4 mainly represented by $\mathrm{T}>\mathrm{C}$ mutation, was similar to 209 COSMIC signature 5 and 16 (cosine similarity 0.868 and 0.9 , respectively. Signature W5 210 displayed high similarity with COSMIC signature 2 and 13 (cosine similarity 0.811 and 0.835 , 211 respectively), which was characterized by $\mathrm{C}>\mathrm{T}$ and $\mathrm{C}>\mathrm{G}$ mutations. COSMIC signatures 2 and 21213 were assigned to the hyperactivity of the APOBEC family enzyme, cytidine deaminases 213 (Alexandrov et al., 2013). One of our samples, OK101, was basaloid squamous cell carcinoma of 214 esophagus, a rare type of malignancy, and a hypermutator in our cohort (Fig. 2G). Somatic 215 SNVs of this sample were dominated by the Signature W5. Signature W6 was characterized by 216 CpTpT-to-CpGpT mutations and highly similar to COSMIC signature 17 (cosine similarity 
217 0.945). COSMIC signature 17 is often found in esophagus and stomach cancer, but its aetiology

218 is unknown. Signature W3 which was defined by $\mathrm{C}>\mathrm{A}, \mathrm{C}>\mathrm{T}$ and $\mathrm{T}>\mathrm{C}$ mutations appeared to

219 have low similarity with any of the COSMIC signatures (all cosine similarity $<0.7$ ).

220 Since, previous studies did not find signatures associated with smoking alone in ESCC

221 despite smoking being a major risk factor of this cancer(Sawada et al., 2016),(Zhang et al.,

222 2015). We examined the association of the mutation signatures with clinical features in ESCC.

223 We found signature W4 was significantly elevated in the smoking patients compared to non-

224 smoking patients $(P=0.01474, t$-test) (Fig. $2 H)$. Mutations of W4 may be caused by the

225 carcinogenic chemical in tobacco smoke. Signature W4 shared similarity with COSMIC

226 signature 5 and 16 . Smoking associated mutational signatures were found higher in the squamous

227 cell carcinomas of lung and head \& neck(Wang et al., 2019). However, there was no difference

228 in total mutation burden between the smoker and non-smoker group $(P=0.7, t$-test $)$ unlike liver

229 cancer. In liver cancer patients, similar signature showed higher mutation rate in smoker group

230 than the nonsmokers(Alexandrov et al., 2016). The other signatures showed no association with

231 smoking, gender, response to chemotherapy and alcohol drinking status $(P>0.05)$.

232

233 Structural variations in ESCC

234 WGS analysis detected a total of 2,641 SVs in the 20 ESCC samples with an average of 132

235 SVs per tumor. The number of SVs varied, ranging from 0 to 515 across the 20 ESCC cases

236 which shows the heterogeneous nature of tumor genome (Fig. S3). In particular, compared to

237 other ESCC cases OK007 and OK008 had high number of SVs which affected most of their

238 chromosomes, indicating genomic instability in these samples (Fig. $3 \boldsymbol{A}-\boldsymbol{L}$ and $\boldsymbol{F i g}$. S4). The

239 deletions were the most abundant type of SVs across the samples (Fig. $3 \boldsymbol{M}$ ). We found 1,090 
240 (41.27\%) deletions followed by 793 (30.03\%) inversions, 391 (14.80\%) translocations and 367

$241(13.90 \%)$ tandem duplications, respectively.

242 We found numerous cancer associated genes affected by SVs in ESCC (Fig. $3 N$ and Table

243 S4). Consistently with a report on Chinese ESCC(Chang et al., 2017), LRP1B (30\%) and TTC28

$244(30 \%)$ were the most commonly affected genes in Japanese ESCC. The tumor suppressor gene

$245 L R P 1 B$ was mostly affected by deletions, whereas TTC28 was by interchromosomal

246 translocations. We were also able to identify $S D K 1$, a novel gene, affected by SVs in $25 \%$ of the

247 ESCC samples. SDK1 is a cell adhesion molecule that plays an active role in cancer

248 development. Somatic mutations in this gene was reported in adrenocortical carcinoma(Juhlin et

249 al., 2015). Recurrent SVs in CSMD1, WWOX, ERC1, PDE4D and SHANK2 were also identified

250 in five tumor samples. The CUB and Sushi multiple domains 1 (CSMD1) is a tumor suppressor

251 gene reported to be associated with poor prognosis in many cancer types including breast cancer,

252 gastric cancer, head and neck squamous cell carcinoma (HNSCC), and hepatocellular

253 carcinoma(Deng et al., 2012),(Zhang et al., 2019),(Jung et al., 2018). Deletion of $W W O X$, a

254 tumor suppressor gene, is frequent in esophageal adenocarcinoma (32\%) and stomach

255 adenocarcinoma (30.2\%) and also observed in other human cancer types such as colon

256 adenocarcinoma, bladder urothelial carcinoma and lung adenocarcinoma (Hussain et al., 2019).

257 Structural rearrangements of ERC1 was previously reported in Chinese ESCC(Chang et al.,

258 2017). ERC1 was also found as a prognostic biomarker in HNSCC (Szczepanski et al., 2013).

259 Previously, a genome wide association study in Chinese Han population identified that SNP

260 rs10052657 in PDE4D on 5q11 was associated with ESCC risk(Wu et al., 2011).

261 Homozygous deletion of $P D E 4 D$ was also identified in breast, lung and gastric cancers which

262 established it as a tumor-promoting gene(Lin et al., 2013). 
Breakage-Fusion-Bridge (BFB) is a mechanism supported by previous studies in

265

266

267

268

269

270

271

272

273

274

275

276

27 278

279

280

281

282

283

284

285

cancer(Cheng et al., 2016)'(Yang et al., 2017). The BFB event is characterized by a special type of structural rearrangements called 'fold-back' inversion. Fold-back inversion can be defined as somatic structural variants with single inverted breakpoints exhibiting copy-number changes(Campbell et al., 2010). We implemented these information to identify BFB in each ESCC genome. In total, we detected 101 fold-back inversions across the 20 ESCCs, of which chromosome 11 appeared to have highest fold-back inversions (30) (Fig. S5A). BFB event was present in total 14 ESCC cases (70\%) in this study. Moreover, fold-back inversions were observed on chromosome 11 around amplification of CCND1 locus (69455873-69469242) in eight ESCC cases (Fig. S5B ). Notably, our CNA analysis identified amplification of CCND1 in fourteen patients, and $57 \%$ of those amplifications (8/14) were caused as a result of BFB. In addition, oncogenes such as FGFRI (1/20) (Fig. S5C) and EGFR (1/20) were also detected in the amplified regions which were affected by BFB event. In all, this analysis presented an important insight of BFB in ESCC, and targeting the amplified oncogenes in therapies will benefit the ESCC patients in future.

Kataegis loci, which are localized hyper-mutation clusters, were identified in $30 \%$ of the ESCC patients (6/20) (Fig. S6A and Table S5). In total 11 kataegis loci were detected in six cases, of which four kataegis had SVs in their close vicinity. Previous studies reported kataegis in ESCC(Chang et al., 2017),(Cheng et al., 2016), and in breast cancer it was observed in more than $50 \%$ of the cases(Nik-Zainal et al., 2012),(D’Antonio et al., 2016). Furthermore, chromothripsis(Korbel \& Campbell, 2013), a phenomenon that affects chromosomes with more than ten structural rearrangements with regular oscillation of copy number changes, was found in 
286

287

288

289

290

291

292

293

294

295

296

297

298

299

300

301

302

303

304

305

306

307 308

one ESCC patient (OK008) (Fig. S6B) on chromosome 12 and 14. Chromothripsis lead structural rearrangements have been reported in many cancers usually in low frequency, however, more than $40 \%$ chromothripsis were found in glioblastomas and lung adenocarcinomas(Korbel \& Campbell, 2013)'(Cortes-Ciriano et al., 2020).

\section{Somatic copy number alterations in ESCC}

We called the somatic CNAs from the whole genome of the 20 pairs of matched tumor and normal samples to investigate CNAs in ESCC. GISTIC2.0 was then used to identify the recurrently amplified and deleted regions(Mermel et al., 2011). We identified four frequent amplified regions (3q26.33, 8p11.23, 11q13.3 and 14q21.1) and six deleted regions (1q21.1, 4q35.2, 5q13.2, 9p21.3, 10p12.33 and 21p11.2) (Fig. $4 \boldsymbol{A}$ and B, Tables S6 and S7).

The copy number amplification of $3 q 26.33$ was found in $63 \%$ of the samples and included important caner driver genes PIK3CA, SOX2, FGF12 and TP63. Notably, SVs in TP63 was also found in $15 \%$ of the ESCC samples in this study. It was observed that $11 q 13.3$ was the most frequently amplified region (74\%) in our dataset, consistently with the observation in Chinese ESCC(Ying et al., 2012). 11q13.3 gain harbored many important genes such as CCND1, FGF3, FGF4 and FGF19 which established this region as a prominent target in ESCC. Amplification of $8 p 11.23$ involved FGFR1, which plays an active role in cell growth and differentiation. Earlier studies reported FGFR1 as a potential drug target in many human cancers(von Loga et al., 2015)'(Chang et al., 2014),(Lin et al., 2014).

Copy number deletion of $9 p 21.3$ was found in $79 \%$ of the samples and it was the most common deletion in the ESCC. This region contains $C D K N 2 A$, an essential regulator of cell cycle (Fig. 4 C). 10p12.33 was deleted in $79 \%$ of the samples and harbored gene $M R C 1$, a M2 
309

310

311

312

313

314 315 316 317 318

319

320

321

322 323 324 325 326 327 328 329 330 331

.

macrophage antigen known to be associated with tumor development, invasion, metastasis and angiogenesis(Weber et al., 2016),(Fang et al., 2017). Deletion of $4 q 35.2$ was found in $63 \%$ of the samples. This region includes the tumor suppressor FAT1, which was recurrently deleted in colorectal cancers, glioblastoma and HNSCC(Morris et al., 2013). Notably, recurrent SNVs/INDELs in FAT1 was observed in our study as well. Overall, this study detected many copy number altered peaks and important ESCC-associated driver genes such as FGFRl, $P I K 3 C A, C C N D 1, C D K N 2 A$ and $M R C 1$, which have the potential to be used as therapeutic targets in future (Du et al., 2017),(Lin et al., 2014),(Padhi et al., 2017),(Weber et al., 2016),(Weber et al., 2014).

\section{Assessment of druggable genes in ESCC}

In order to examine the druggability of the genes identified by the CNA analysis, we analyzed the genes with the drug-gene interaction database(Griffith et al., 2013),(Cotto et al., 2018). We found that 67 genes out of the 442 genes had at least one drug target that interact with it (Table S8). HTR1A, PIK3CA, FGFR1, ADRB3, HPIK3R1, MTNR1A, CDKN2A, CCND1, $C D K 7$, and $A N O 1$ were the top druggable genes that showed large interactions with drugs. Notably, amplification of CCND1 (74\%), PIK3CA (63\%), FGFR1 (37\%) and deletion of CDKN2A (79\%) and CDK7 (74\%) genes were observed in a considerable amount of samples in our analysis. In all, CNA analysis was able to identify potentially druggable genes including CCND1, FGFR1, PIK3CA and CDKN2A which might be used for the treatment of ESCC in future.

The druggability of genes detected by our SNV analysis was also examined. We identified 212 genes out of the 1,111 genes that have at least one interaction with drug (Table 
332 S9). We identified PIK3CA, TP53, BRAF, NOTCH1, FGFR2, F11, MTOR, LRP2, and RB1 were

333 the most prominent druggable genes with large number of drug-interactions. On the other hand,

334149 genes out of 958 have the druggable property which were identified by SV analysis here

335 (Table S10). LRP1B, PDE4D, WWOX, GPHN, KCNB2, FHIT, CDKN2A, TP53 and BRCA2

336 were the important druggable genes determined with large number of interactions with drugs.

337 LRP1B (30\%), PDE4D (25\%), WWOX (25\%), GPHN (20\%) and KCNB2 (20\%) were frequently 338 affected by SVs in ESCC.

339 We also combined the analysis of druggable genes identified by all the mutational events

340 SNVs, SVs and CNAs in ESCC. It was observed that some of the genes such as TP53, NOTCH1,

$341 R B 1, J A K 2, A C A N$ and SCN9A were commonly altered by both SNVs and SVs in ESCC (Fig. 5

342 and Table S11). Genes, for example, PIK3CA, F11, EPHB3 and TLR3 were commonly altered

343 by SNVs and CNAs. We also detected some genes such as CDKN2A, ANO1 and NDUFB5 were

344 altered by both SVs and CNAs. However, we found no druggable gene that was altered

345 commonly by all the three mutation patterns in our analysis.

346

\section{Discussion}

In this study, we performed a comprehensive whole genome sequencing analysis in order

349 to explore mutational landscape in ESCC with Japanese origin. Previously, several studies

350 reported mutations in ESCC mostly using whole exome data, which is limited to coding

351 mutations. Here, we provided a complete whole-genome level analysis of mutational signatures,

352 SNVs, SVs and CNAs in ESCC. Consistent with the previous studies, this study also confirmed

353 the frequent mutation of TP53, ZNF750, FAT1, PTCH1, EP300, FAT2, FBXW7, KMT2D,

354 NFE2L2, NOTCH1, PIK3CA, RB1, RIPK4 and TP63 in ESCC patients (Chang et al., 
355

356

357

358

359

360

361

362

363

364

365

366

367 368

369

370

371

372

373

374

375

376

377

2017),(Sawada et al., 2016),(Qin et al., 2016). Recurrent mutations in most of these genes were

previously reported in HNSCC(Lin et al., 2018),(Hazawa et al., 2017),(TCGA, 2015) and lung squamous cell carcinoma(Choi et al., 2017),(Li et al., 2015).

By analyzing mutational signatures we showed distinct signature profiles in ESCC and their correlation with environmental risk factor and clinical features. Importantly, among the identified six mutational signatures, significant association was shown between signature W4 and smoking status of ESCC patients. This signature W4 showed higher similarity with COSMIC signature 5 and 16 (cosine similarity 0.868 and 0.9 , respectively). A WGS study showed association of COSMIC signature 16 with alcohol drinking and smoking status in ESCC patients of Chinese origin(Chang et al., 2017). Smoking tobacco increases the chances of cancer development. Tobacco smoke is composed of many carcinogens which are capable enough to disrupt the DNA. Tobacco smoking has been linked to many type of cancers such as lung cancer, liver cancer, esophageal cancer and gastric cancer(Alexandrov et al., 2016). COSMIC Signature 5, which is also characterized by $\mathrm{C}>\mathrm{T}$ and $\mathrm{T}>\mathrm{C}$ mutations, was reported in smoking associated lung cancer. Smoking associated signature was more commonly found in male patients (65\%) with smoking history than female, and $62 \%$ of those patients were non-responder to chemotherapy. In contrast, the female patients (30\%) who showed this signature were mostly non-smoker $(86 \%)$. However, the female patients with nonsmoking history who showed signature $\mathrm{W} 4,84 \%$ of them responded well to the chemotherapy.

A recent WGS analysis on UK patients suggested $\mathrm{C}>\mathrm{A} / \mathrm{T}$ as a distinct mutational pattern with evidence of ageing in EAC(Secrier et al., 2016). We confirmed that $\mathrm{C}>\mathrm{T}$ and $\mathrm{T}>\mathrm{C}$ substitutions were also dominant type mutations with age and smoking imprint in ESCC as well. Further, it was previously described that these mutation patterns characterize the smoking and 
378 alcohol drinking signatures by Alexandrov et al.(Alexandrov et al., 2016). On the other hand, 379 mutational signatures identified here showed high similarity not only with COSMIC signatures 380 associated with alcohol drinking and smoking but also with age and APOBEC signature activity 381 related signatures. APOBEC family enzymes alter cytidine to uracil through the process of 382 deamination within DNA which leads to mutation clusters in different cancers(Harris, Petersen383 Mahrt \& Neuberger, 2002). Previous studies reported the association of PIK3CA mutations with 384 APOBEC signature in Chinese and Japanese ESCC(Chang et al., 2017).(Sawada et al., 385 2016)'(Zhang et al., 2015). In this study, we found that APOBEC signature is positively 386 associated $(P=0.00003969$, Wilcoxon rank sum test $)$ with amplification of PIK3CA. APOBEC 387 signature was observed in all the ESCC tumor samples (100\%). APOBEC mediated genomic 388 damages may play a major role in ESCC development.

389 In the present study, we analyzed SVs using WGS of 20 ESCCs which was the first time 390 report in Japanese population. A recent analysis of WGS of 94 ESCCs from China showed $391 L R P 1 B$ and TTC28 as the most commonly affected genes by SVs(Chang et al., 2017). We 392 confirmed this finding in Japanese ESCCs as well. $L R P 1 B$ was mostly affected by deletions and 393 TTC28 by interchromosomal translocation in all the cases. Further, this analysis identified SVs in 394 a novel gene SDK1 in 5 out of the 20 ESCC tumor samples. An epigenomic study identified $395 S D K 1$ as an epigenomic driver in hepatocellular carcinoma(Gentilini et al., 2017). Besides, SVs 396 were found in other genes such as WWOX, CSMD1, ERC1, PDE4D, SHANK2, and TP63. This 397 study found two patterns of structural rearrangements across the 20 ESCC genome. In the first 398 pattern samples were found with a few rearrangements and in the other, samples were with 399 multiple complex rearrangements which represent the heterogeneous nature of ESCCs.

400 Moreover, this study identified breakage-fusion-bridge, kataegis and chromothripsis in $75 \%$ of 
401 the ESCC patients (15/20). Chromothripsis, a phenomenon when multiple rearrangements affect 402 a single or multiple chromosomes in a single event (Rode et al., 2016),(Korbel \& Campbell, 403 2013). It is believed that chromothripsis plays important role in the development of cancer. In 404 recent years, chromothripsis has emerged as a significant biomarker in different cancers. 405 Chromothripsis may promote cancer development either by increasing copy numbers of 406 oncogenes or by deleting important tumor suppressors. Thus, identification of chromothripsis 407 occurrence in tumors using strict criteria is crucial for cancer genomics. More studies are 408 required with a comparatively larger sample size in future.

409 Moreover, we identified multiple significantly amplified and deleted regions in ESCC 410 genomes. These regions harbored many genes that may be used as therapeutic targets. 411 Amplification of 11 1 13.3 region, which contains CCND1, CTTN, SHANK2 and three FGF412 family genes, was frequently altered in patients that responded to chemotherapy. CCND1, a key 413 regulator of the G1 phase of cell cycle, is an important target of chemotherapy response in 414 HNSCC(Feng et al., 2011). It has been associated with poor prognosis in many solid cancers 415 including HNSCC(Feng et al., 2011). Another amplification region 3q26.33 harbored important 416 caner-causing genes such as PIK3CA, SOX2, FGF12 and TP63. Alterations of PIK3CA which 417 leads to dysfunction of cell cycle control, was also frequently found in colorectal cancer, 418 HNSCC, gastric cancer and breast cancer(Mei et al., 2016),(De Mello et al., 2018),(Azizi Tabesh 419 et al., 2017),(Du et al., 2017). PIK3CA was reported to have association with drug sensitivity in 420 many cancer types including ESCC(Du et al., 2017),(Yokota et al., 2018). Furthermore, 421 amplification of TP63 was also commonly found in squamous cell carcinomas of lung, and neck 422 and head which showed over-expression in these tumors(TCGA, 2015),(Ohnami et al., 2017). 423 TP63 is a transcription factor and plays important role in tumorigenesis, apoptosis and 
424 embryogenesis(Ohnami et al., 2017),(Candi et al., 2014). Deletion of CDKN2A gene is more

425 frequent in Japanese than Chinese ESCC. We confirmed deletion of CDKN2A in $9 p 21.3$ and $426 \mathrm{MRC1}$ in $10 p 12.33$ regions as the most common deletion (79\%) in our dataset. Previously 427 several studies reported recurrent mutations and loss in $C D K N 2 A$ in many human cancer types 428 such as pancreatic cancer and oral squamous cell carcinoma(Zhen et al., 2015)'(Padhi et al., 429 2017). A previous study on Chinese population also reported deletion of $C D K N 2 A$, but only $30 \%$ 430 of the Chinese samples showed deletion of this gene. Our gene-drug interaction analysis was also 431 able to detect druggable genes for example FGFR1, PIK3CA,CCND1, CDKN2A, CDK7 and 432 ANO1 that have the potential to interact with at least one drug.

433 There were some limitations in this study. The main bottle-neck of this study was the sample 434 size. We had 20 pairs of matched normal and tumor samples, which was not enough to find 435 significantly mutated recurrent genes with high level of frequencies. Secondly, although the 436 patient's chemotherapy response information was available, the small number of sample size did 437 not allow us to associate the identified genomic alterations with chemotherapy response at a 438 significant level. It would be important to associate the genomic alterations with chemotherapy 439 response to select the correct patients for effective neoadjuvent chemotherapy in future.

\section{Conclusions}

442 In summary, this analysis was able to identify the frequent genomic mutations, mutational 443 signatures and significant association with environmental risk factor, druggable CNAs and 444 genes, and structural rearrangements in ESCC. This comprehensive analysis provided insights 445 into the ESCC development at molecular level and identified targets for the diagnosis and 446 treatment of ESCC in future. 


\section{Acknowledgements}

449 The super-computing resource 'SHIROKANE' was provided by the Human Genome Center, 450 The University of Tokyo (http://supcom.hgc.jp/).

451

452

453

454

455

456

457

458

459

460

461

462

463

464

465

466

467

468

469

\section{References}

Alexandrov LB, Ju YS, Haase K, Van Loo P, Martincorena I, Nik-Zainal S, Totoki Y, Fujimoto A, Nakagawa H, Shibata T, Campbell PJ, Vineis P, Phillips DH, Stratton MR. 2016. Mutational signatures associated with tobacco smoking in human cancer. Science (New York, N.Y.) 354:618-622. DOI: 10.1126/science.aag0299.

Alexandrov LB, Nik-Zainal S, Wedge DC, Aparicio SAJR, Behjati S, Biankin A V, Bignell GR, Bolli N, Borg A, Borresen-Dale A-L, Boyault S, Burkhardt B, Butler AP, Caldas C, Davies HR, Desmedt C, Eils R, Eyfjord JE, Foekens JA, Greaves M, Hosoda F, Hutter B, Ilicic T, Imbeaud S, Imielinski M, Jager N, Jones DTW, Jones D, Knappskog S, Kool M, Lakhani SR, Lopez-Otin C, Martin S, Munshi NC, Nakamura H, Northcott PA, Pajic M, Papaemmanuil E, Paradiso A, Pearson J V, Puente XS, Raine K, Ramakrishna M, Richardson AL, Richter J, Rosenstiel P, Schlesner M, Schumacher TN, Span PN, Teague JW, Totoki Y, Tutt ANJ, Valdes-Mas R, van Buuren MM, van ’t Veer L, Vincent-Salomon A, Waddell N, Yates LR, Zucman-Rossi J, Futreal PA, McDermott U, Lichter P, Meyerson M, Grimmond SM, Siebert R, Campo E, Shibata T, Pfister SM, Campbell PJ, Stratton MR. 2013. Signatures of mutational processes in human cancer. Nature 500:415-421. DOI: 10.1038/nature12477. 
470 Azizi Tabesh G, Izadi P, Fereidooni F, Emami Razavi AN, Tavakkoly Bazzaz J. 2017. The High

471 Frequency of PIK3CA Mutations in Iranian Breast Cancer Patients. Cancer investigation

472 35:36-42. DOI: 10.1080/07357907.2016.1247455.

473 Baba Y, Watanabe M, Yoshida N, Baba H. 2014. Neoadjuvant treatment for esophageal

474 squamous cell carcinoma. World journal of gastrointestinal oncology 6:121-128. DOI:

$475 \quad$ 10.4251/wjgo.v6.i5.121.

476 Campbell PJ, Yachida S, Mudie LJ, Stephens PJ, Pleasance ED, Stebbings LA, Morsberger LA,

477 Latimer C, McLaren S, Lin M-L, McBride DJ, Varela I, Nik-Zainal SA, Leroy C, Jia M,

478 Menzies A, Butler AP, Teague JW, Griffin CA, Burton J, Swerdlow H, Quail MA, Stratton

479 MR, Iacobuzio-Donahue C, Futreal PA. 2010. The patterns and dynamics of genomic

480 instability in metastatic pancreatic cancer. Nature 467:1109-1113. DOI:

$481 \quad 10.1038 /$ nature09460.

482 Candi E, Agostini M, Melino G, Bernassola F. 2014. How the TP53 family proteins TP63 and

483 TP73 contribute to tumorigenesis: regulators and effectors. Human mutation 35:702-714.

484 DOI: 10.1002/humu.22523.

485 Chang J, Liu X, Wang S, Zhang Z, Wu Z, Zhang X, Li J. 2014. Prognostic value of FGFR gene

486 amplification in patients with different types of cancer: a systematic review and meta-

487 analysis. PloS one 9:e105524. DOI: 10.1371/journal.pone.0105524.

488 Chang J, Tan W, Ling Z, Xi R, Shao M, Chen M, Luo Y, Zhao Y, Liu Y, Huang X, Xia Y, Hu J,

489 Parker JS, Marron D, Cui Q, Peng L, Chu J, Li H, Du Z, Han Y, Tan W, Liu Z, Zhan Q, Li

490 Y, Mao W, Wu C, Lin D. 2017. Genomic analysis of oesophageal squamous-cell carcinoma

491 identifies alcohol drinking-related mutation signature and genomic alterations. Nature

492 communications 8:15290. DOI: 10.1038/ncomms 15290. 
493 Chattopadhyay I, Singh A, Phukan R, Purkayastha J, Kataki A, Mahanta J, Saxena S, Kapur S.

494 2010. Genome-wide analysis of chromosomal alterations in patients with esophageal

495 squamous cell carcinoma exposed to tobacco and betel quid from high-risk area in India.

496 Mutation research 696:130-138. DOI: 10.1016/j.mrgentox.2010.01.001.

497 Cheng C, Zhou Y, Li H, Xiong T, Li S, Bi Y, Kong P, Wang F, Cui H, Li Y, Fang X, Yan T, Li

498 Y, Wang J, Yang B, Zhang L, Jia Z, Song B, Hu X, Yang J, Qiu H, Zhang G, Liu J, Xu E,

499 Shi R, Zhang Y, Liu H, He C, Zhao Z, Qian Y, Rong R, Han Z, Zhang Y, Luo W, Wang J,

500 Peng S, Yang X, Li X, Li L, Fang H, Liu X, Ma L, Chen Y, Guo S, Chen X, Xi Y, Li G,

501 Liang J, Yang X, Guo J, Jia J, Li Q, Cheng X, Zhan Q, Cui Y. 2016. Whole-Genome

502 Sequencing Reveals Diverse Models of Structural Variations in Esophageal Squamous Cell

503 Carcinoma. American journal of human genetics 98:256-274. DOI:

$504 \quad$ 10.1016/j.ajhg.2015.12.013.

505 Choi M, Kadara H, Zhang J, Parra ER, Rodriguez-Canales J, Gaffney SG, Zhao Z, Behrens C,

506 Fujimoto J, Chow C, Kim K, Kalhor N, Moran C, Rimm D, Swisher S, Gibbons DL,

507 Heymach J, Kaftan E, Townsend JP, Lynch TJ, Schlessinger J, Lee J, Lifton RP, Herbst RS,

508 Wistuba II. 2017. Mutation profiles in early-stage lung squamous cell carcinoma with

509 clinical follow-up and correlation with markers of immune function. Annals of oncology :

$510 \quad$ official journal of the European Society for Medical Oncology 28:83-89. DOI:

$511 \quad$ 10.1093/annonc/mdw437.

512 Cortes-Ciriano I, Lee JJ-K, Xi R, Jain D, Jung YL, Yang L, Gordenin D, Klimczak LJ, Zhang C-

513 Z, Pellman DS, Park PJ. 2020. Comprehensive analysis of chromothripsis in 2,658 human

514 cancers using whole-genome sequencing. Nature genetics. DOI: 10.1038/s41588-019-0576-

$515 \quad 7$. 
516 Cotto KC, Wagner AH, Feng Y-Y, Kiwala S, Coffman AC, Spies G, Wollam A, Spies NC, 517 Griffith OL, Griffith M. 2018. DGIdb 3.0: a redesign and expansion of the drug-gene 518 interaction database. Nucleic acids research 46:D1068-D1073. DOI: 10.1093/nar/gkx1143. 519 D’Antonio M, Tamayo P, Mesirov JP, Frazer KA. 2016. Kataegis Expression Signature in Breast 520 Cancer Is Associated with Late Onset, Better Prognosis, and Higher HER2 Levels. Cell 521 reports 16:672-683. DOI: 10.1016/j.celrep.2016.06.026.

522 Deng N, Goh LK, Wang H, Das K, Tao J, Tan IB, Zhang S, Lee M, Wu J, Lim KH, Lei Z, Goh 523 G, Lim Q, Tan AL, Yu D, Poh S, Riahi S, Bell S, Shi MM, Linnartz R, Cheong HC, Rha 524 SY, Boussioutas A, Grabsch H. 2012. A comprehensive survey of genomic alterations in 525 gastric cancer reveals systematic patterns of molecular exclusivity and co-occurrence 526 among distinct therapeutic targets. DOI: 10.1136/gutjnl-2011-301839.

527 528 529 530 531 532 533 534 535 536 537 538

Du P, Huang P, Huang X, Li X, Feng Z, Li F, Liang S, Song Y, Stenvang J, Brunner N, Yang H, Ou Y, Gao Q, Li L. 2017. Comprehensive genomic analysis of Oesophageal Squamous Cell Carcinoma reveals clinical relevance. Scientific reports 7:15324. DOI: 10.1038/s41598017-14909-5.

Fang J, Li X, Ma D, Liu X, Chen Y, Wang Y, Lui VWY, Xia J, Cheng B, Wang Z. 2017. Prognostic significance of tumor infiltrating immune cells in oral squamous cell carcinoma. BMC cancer 17:375. DOI: 10.1186/s12885-017-3317-2.

Feng Z, Guo W, Zhang C, Xu Q, Zhang P, Sun J, Zhu H, Wang Z, Li J, Wang L, Wang B, Ren G, Ji T, Tu W, Yang X, Qiu W, Mao L, Zhang Z, Chen W. 2011. CCND1 as a Predictive Biomarker of Neoadjuvant Chemotherapy in Patients with Locally Advanced Head and Neck Squamous Cell Carcinoma. 6. DOI: 10.1371/journal.pone.0026399.

Fujimoto A, Furuta M, Totoki Y, Tsunoda T, Kato M, Shiraishi Y, Tanaka H, Taniguchi H, 
Kawakami Y, Ueno M, Gotoh K, Ariizumi S-I, Wardell CP, Hayami S, Nakamura T,

540

541

542

543

544

545

546

547

548

549

550

551

552

553

554

555

556

557

558

559

560

Aikata H, Arihiro K, Boroevich KA, Abe T, Nakano K, Maejima K, Sasaki-Oku A, Ohsawa A, Shibuya T, Nakamura H, Hama N, Hosoda F, Arai Y, Ohashi S, Urushidate T, Nagae G, Yamamoto S, Ueda H, Tatsuno K, Ojima H, Hiraoka N, Okusaka T, Kubo M, Marubashi S, Yamada T, Hirano S, Yamamoto M, Ohdan H, Shimada K, Ishikawa O, Yamaue H, Chayama K, Miyano S, Aburatani H, Shibata T, Nakagawa H. 2016. Whole-genome mutational landscape and characterization of noncoding and structural mutations in liver cancer. Nature genetics 48:500-509. DOI: 10.1038/ng.3547.

Gentilini D, Scala S, Gaudenzi G, Garagnani P, Capri M, Cescon M, Grazi GL, Bacalini MG, Pisoni S, Dicitore A, Circelli L, Santagata S, Izzo F, Di Blasio AM, Persani L, Franceschi C, Vitale G. 2017. Epigenome-wide association study in hepatocellular carcinoma: Identification of stochastic epigenetic mutations through an innovative statistical approach. Oncotarget 8:41890-41902. DOI: 10.18632/oncotarget.17462.

Griffith M, Griffith OL, Coffman AC, Weible J V, McMichael JF, Spies NC, Koval J, Das I, Callaway MB, Eldred JM, Miller CA, Subramanian J, Govindan R, Kumar RD, Bose R, Ding L, Walker JR, Larson DE, Dooling DJ, Smith SM, Ley TJ, Mardis ER, Wilson RK. 2013. DGIdb: mining the druggable genome. Nature methods 10:1209-1210. DOI: 10.1038/nmeth.2689.

Harris RS, Petersen-Mahrt SK, Neuberger MS. 2002. RNA editing enzyme APOBEC1 and some of its homologs can act as DNA mutators. Molecular cell 10:1247-1253. DOI: $10.1016 / \mathrm{s} 1097-2765(02) 00742-6$.

Hazawa M, Lin D-C, Handral H, Xu L, Chen Y, Jiang Y-Y, Mayakonda A, Ding L-W, Meng X, Sharma A, Samuel S, Movahednia MM, Wong RW, Yang H, Tong C, Koeffler HP. 2017. 
562

563

564

565

566

567

568

569

570

571

572

573

574

575

576

577

578

579

580

581

582

583

584

ZNF750 is a lineage-specific tumour suppressor in squamous cell carcinoma. Oncogene 36:2243-2254. DOI: 10.1038/onc.2016.377.

Hermetz KE, Newman S, Conneely KN, Martin CL, Ballif BC, Shaffer LG, Cody JD, Rudd MK. 2014. Large inverted duplications in the human genome form via a fold-back mechanism. PLoS genetics 10:e1004139. DOI: 10.1371/journal.pgen.1004139.

Hussain T, Liu B, Shrock MS, Williams T, Aldaz CM. 2019. WWOX, the FRA16D gene: A target of and a contributor to genomic instability. Genes, chromosomes \& cancer 58:324338. DOI: $10.1002 /$ gcc.22693.

Juhlin CC, Goh G, Healy JM, Fonseca AL, Scholl UI, Stenman A, Kunstman JW, Brown TC, Overton JD, Mane SM, Nelson-Williams C, Backdahl M, Suttorp A-C, Haase M, Choi M, Schlessinger J, Rimm DL, Hoog A, Prasad ML, Korah R, Larsson C, Lifton RP, Carling T. 2015. Whole-exome sequencing characterizes the landscape of somatic mutations and copy number alterations in adrenocortical carcinoma. The Journal of clinical endocrinology and metabolism 100:E493-502. DOI: 10.1210/jc.2014-3282.

Jung AR, Eun Y-G, Lee YC, Noh JK, Kwon KH. 2018. Clinical Significance of CUB and Sushi Multiple Domains 1 Inactivation in Head and Neck Squamous Cell Carcinoma. International journal of molecular sciences 19. DOI: 10.3390/ijms19123996.

Koboldt DC, Zhang Q, Larson DE, Shen D, Mclellan MD, Lin L, Miller CA, Mardis ER, Ding L, Wilson RK. 2012. VarScan 2 : Somatic mutation and copy number alteration discovery in cancer by exome sequencing. :568-576. DOI: 10.1101/gr.129684.111.568.

Korbel JO, Campbell PJ. 2013. Criteria for inference of chromothripsis in cancer genomes. Cell 152:1226-1236. DOI: 10.1016/j.cell.2013.02.023.

Li C, Gao Z, Li F, Li X, Sun Y, Wang M, Li D, Wang R, Li F, Fang R, Pan Y, Luo X, He J, 
585

586

587

588

589

590

591

592

593

594

595

596

597

598

599

600

601

602

603

604

605

606

607

Zheng L, Xia J, Qiu L, He J, Ye T, Zhang R, He M, Zhu M, Hu H, Shi T, Zhou X, Sun M, Tian S, Zhou Y, Wang Q, Chen L, Yin G, Lu J, Wu R, Guo G, Li Y, Hu X, Li L, Asan, Wang Q, Yin Y, Feng Q, Wang B, Wang H, Wang M, Yang X, Zhang X, Yang H, Jin L, Wang C-Y, Ji H, Chen H, Wang J, Wei Q. 2015. Whole Exome Sequencing Identifies Frequent Somatic Mutations in Cell-Cell Adhesion Genes in Chinese Patients with Lung Squamous Cell Carcinoma. Scientific reports 5:14237. DOI: 10.1038/srep14237.

Li XC, Wang MY, Yang M, Dai HJ, Zhang BF, Wang W, Chu XL, Wang X, Zheng H, Niu RF, Zhang W, Chen KX. 2018. A mutational signature associated with alcohol consumption and prognostically significantly mutated driver genes in esophageal squamous cell carcinoma Original article. 2:938-944. DOI: 10.1093/annonc/mdy011.

Lin D, Hao J, Nagata Y, Xu L, Shang L, Meng X, Sato Y, Okuno Y, Varela AM, Ding L, Garg M, Liu L, Yang H, Yin D, Shi Z, Jiang Y, Gu W, Gong T, Zhang Y, Xu X, Kalid O, Shacham S, Ogawa S, Wang M, Koeffler HP. 2014. Genomic and molecular characterization of esophageal squamous cell carcinoma. Nature Publishing Group 46:467473. DOI: $10.1038 / \mathrm{ng} .2935$.

Lin S-C, Lin L-H, Yu S-Y, Kao S-Y, Chang K-W, Cheng H-W, Liu C-J. 2018. FAT1 somatic mutations in head and neck carcinoma are associated with tumor progression and survival. Carcinogenesis 39:1320-1330. DOI: 10.1093/carcin/bgy107.

Lin D-C, Xu L, Ding L-W, Sharma A, Liu L-Z, Yang H, Tan P, Vadgama J, Karlan BY, Lester J, Urban N, Schummer M, Doan N, Said JW, Sun H, Walsh M, Thomas CJ, Patel P, Yin D, Chan D, Koeffler HP. 2013. Genomic and functional characterizations of phosphodiesterase subtype 4D in human cancers. Proceedings of the National Academy of Sciences of the United States of America 110:6109-6114. DOI: 10.1073/pnas.1218206110. 
608 von Loga K, Kohlhaussen J, Burkhardt L, Simon R, Steurer S, Burdak-Rothkamm S, Jacobsen F, 609 Sauter G, Krech T. 2015. FGFR1 Amplification Is Often Homogeneous and Strongly 610 Linked to the Squamous Cell Carcinoma Subtype in Esophageal Carcinoma. PloS one 611 10:e0141867. DOI: 10.1371/journal.pone.0141867.

612 Martincorena I, Raine KM, Gerstung M, Dawson KJ, Haase K, Van Loo P, Davies H, Stratton 613 MR, Campbell PJ. 2017. Universal Patterns of Selection in Cancer and Somatic Tissues. 614 Cell 171:1029-1041.e21. DOI: 10.1016/j.cell.2017.09.042.

615 Mayakonda A, Lin D-C, Assenov Y, Plass C, Koeffler HP. 2018. Maftools: efficient and 616 comprehensive analysis of somatic variants in cancer. Genome research 28:1747-1756. 617 DOI: $10.1101 /$ gr.239244.118.

618 Mei ZB, Duan CY, Li CB, Cui L, Ogino S. 2016. Prognostic role of tumor PIK3CA mutation in 619 colorectal cancer: a systematic review and meta-analysis. Annals of oncology : official 620 journal of the European Society for Medical Oncology 27:1836-1848. DOI:

621 10.1093/annonc/mdw264.

622 De Mello RA, Castelo-Branco L, Castelo-Branco P, Pozza DH, Vermeulen L, Palacio S, 623 Salzberg M, Lockhart AC. 2018. What Will We Expect From Novel Therapies to 624 Esophageal and Gastric Malignancies? American Society of Clinical Oncology educational 625 book. American Society of Clinical Oncology. Annual Meeting 38:249-261. DOI:

626 $10.1200 /$ EDBK_198805.

627 Mermel CH, Schumacher SE, Hill B, Meyerson ML, Beroukhim R, Getz G. 2011. GISTIC2.0 628 facilitates sensitive and confident localization of the targets of focal somatic copy-number 629 alteration in human cancers. Genome biology 12:R41. DOI: 10.1186/gb-2011-12-4-r41. 630 Morris LGT, Kaufman AM, Gong Y, Ramaswami D, Walsh LA, Turcan S, Eng S, Kannan K, 
631

632

633

634

635

636

637

638

639

640

641

642

643

644

645

646

647

648

649

650

651

652

653

Zou Y, Peng L, Banuchi VE, Paty P, Zeng Z, Vakiani E, Solit D, Singh B, Ganly I, Liau L, Cloughesy TC, Mischel PS, Mellinghoff IK, Chan TA. 2013. Recurrent somatic mutation of FAT1 in multiple human cancers leads to aberrant Wnt activation. Nature genetics 45:253261. DOI: $10.1038 / \mathrm{ng} .2538$.

Nik-Zainal S, Alexandrov LB, Wedge DC, Van Loo P, Greenman CD, Raine K, Jones D, Hinton J, Marshall J, Stebbings LA, Menzies A, Martin S, Leung K, Chen L, Leroy C, Ramakrishna M, Rance R, Lau KW, Mudie LJ, Varela I, McBride DJ, Bignell GR, Cooke SL, Shlien A, Gamble J, Whitmore I, Maddison M, Tarpey PS, Davies HR, Papaemmanuil E, Stephens PJ, McLaren S, Butler AP, Teague JW, Jonsson G, Garber JE, Silver D, Miron P, Fatima A, Boyault S, Langerod A, Tutt A, Martens JWM, Aparicio SAJR, Borg A, Salomon AV, Thomas G, Borresen-Dale A-L, Richardson AL, Neuberger MS, Futreal PA, Campbell PJ, Stratton MR. 2012. Mutational processes molding the genomes of 21 breast cancers. Cell 149:979-993. DOI: 10.1016/j.cell.2012.04.024.

Ohnami S, Ohshima K, Nagashima T, Urakami K, Shimoda Y, Saito J, Naruoka A, Hatakeyama K, Mochizuki T, Serizawa M, Ohnami S, Kusuhara M, Yamaguchi K. 2017. Comprehensive characterization of genes associated with the TP53 signal transduction pathway in various tumors. Molecular and cellular biochemistry 431:75-85. DOI: 10.1007/s11010-017-2977-1.

Padhi SS, Roy S, Kar M, Saha A, Roy S, Adhya A, Baisakh M, Banerjee B. 2017. Role of CDKN2A/p16 expression in the prognostication of oral squamous cell carcinoma. Oral oncology 73:27-35. DOI: 10.1016/j.oraloncology.2017.07.030.

Qin H-D, Liao X-Y, Chen Y-B, Huang S-Y, Xue W-Q, Li F-F, Ge X-S, Liu D-Q, Cai Q, Long J, Li X-Z, Hu Y-Z, Zhang S-D, Zhang L-J, Lehrman B, Scott AF, Lin D, Zeng Y-X, Shugart 
654

655

656

657

658

659

660

661

662

663

664

665

666

667

668

669

670

671

672

673

674

675

676

YY, Jia W-H. 2016. Genomic Characterization of Esophageal Squamous Cell Carcinoma Reveals Critical Genes Underlying Tumorigenesis and Poor Prognosis. American journal of human genetics 98:709-727. DOI: 10.1016/j.ajhg.2016.02.021.

Rode A, Maass KK, Willmund KV, Lichter P, Ernst A. 2016. Chromothripsis in cancer cells: An update. International journal of cancer 138:2322-2333. DOI: 10.1002/ijc.29888.

Sawada G, Niida A, Uchi R, Hirata H, Shimamura T, Suzuki Y, Shiraishi Y, Chiba K, Imoto S, Takahashi Y, Iwaya T, Sudo T, Hayashi T, Takai H, Kawasaki Y, Matsukawa T, Eguchi H, Sugimachi K, Tanaka F, Suzuki H, Yamamoto K, Ishii H, Shimizu M, Yamazaki H, Yamazaki M, Tachimori Y, Kajiyama Y, Natsugoe S, Fujita H, Mafune K, Tanaka Y, Kelsell DP, Scott CA, Tsuji S, Yachida S, Shibata T, Sugano S, Doki Y, Akiyama T, Aburatani H, Ogawa S, Miyano S, Mori M, Mimori K. 2016. Genomic Landscape of Esophageal Squamous Cell Carcinoma in a Japanese Population. Gastroenterology 150:1171-1182. DOI: 10.1053/j.gastro.2016.01.035.

Secrier M, Li X, de Silva N, Eldridge MD, Contino G, Bornschein J, MacRae S, Grehan N, O’Donovan M, Miremadi A, Yang T-P, Bower L, Chettouh H, Crawte J, Galeano-Dalmau N, Grabowska A, Saunders J, Underwood T, Waddell N, Barbour AP, Nutzinger B, Achilleos A, Edwards PAW, Lynch AG, Tavare S, Fitzgerald RC. 2016. Mutational signatures in esophageal adenocarcinoma define etiologically distinct subgroups with therapeutic relevance. Nature genetics 48:1131-1141. DOI: 10.1038/ng.3659.

Song Y, Li L, Ou Y, Gao Z, Li E, Li X, Zhang W, Wang J, Xu L, Zhou Y, Ma X, Liu L, Zhao Z, Huang X, Fan J, Dong L, Chen G, Ma L, Yang J, Chen L, He M, Li M, Zhuang X, Huang K, Qiu K, Yin G, Guo G, Feng Q, Chen P, Wu Z, Wu J, Ma L, Zhao J, Luo L, Fu M, Xu B, Chen B, Li Y, Tong T, Wang M, Liu Z, Lin D, Zhang X, Yang H, Wang J, Zhan Q. 2014. 
677 Identification of genomic alterations in oesophageal squamous cell cancer. Nature 509:91678 95. DOI: 10.1038/nature13176.

679 Szczepanski MJ, DeLeo AB, Luczak M, Molinska-Glura M, Misiak J, Szarzynska B, Dworacki 680 G, Zagor M, Rozwadowska N, Kurpisz M, Krzeski A, Kruk-Zagajewska A, Kopec T, 681 Banaszewski J, Whiteside TL. 2013. PRAME expression in head and neck cancer correlates 682 with markers of poor prognosis and might help in selecting candidates for retinoid 683 chemoprevention in pre-malignant lesions. Oral oncology 49:144-151. DOI: $684 \quad$ 10.1016/j.oraloncology.2012.08.005.

685 TCGA. 2015. Comprehensive genomic characterization of head and neck squamous cell $686 \quad$ carcinomas. Nature 517:576-582. DOI: 10.1038/nature14129.

687 TCGA. 2017. Integrated genomic characterization of oesophageal carcinoma. Nature:169-175. 688 DOI: $10.1038 /$ nature20805.

689 Wang K, Li M, Hakonarson H. 2010. ANNOVAR: functional annotation of genetic variants 690 from high-throughput sequencing data. Nucleic acids research 38:e164. DOI:

691 10.1093/nar/gkq603.

692 Wang J, Linxweiler M, Yang W, Chan TA, Morris LGT. 2019. Immunomodulatory and 693 immunotherapeutic implications of tobacco smoking in squamous cell carcinomas and 694 normal airway epithelium. Oncotarget 10:3835-3839. DOI: 10.18632/oncotarget.26982. 695 Weber M, Buttner-Herold M, Hyckel P, Moebius P, Distel L, Ries J, Amann K, Neukam FW, 696 Wehrhan F. 2014. Small oral squamous cell carcinomas with nodal lymphogenic metastasis 697 show increased infiltration of M2 polarized macrophages--an immunohistochemical 698 analysis. Journal of cranio-maxillo-facial surgery : official publication of the European 699 Association for Cranio-Maxillo-Facial Surgery 42:1087-1094. DOI: 
700

701

702

703

704

705

706

707

708

709

710

711

712

713

714

715

716

717

718

719

720

721

722

10.1016/j.jcms.2014.01.035.

Weber M, Iliopoulos C, Moebius P, Buttner-Herold M, Amann K, Ries J, Preidl R, Neukam FW, Wehrhan F. 2016. Prognostic significance of macrophage polarization in early stage oral squamous cell carcinomas. Oral oncology 52:75-84. DOI:

10.1016/j.oraloncology.2015.11.001.

Wu C, Hu Z, He Z, Jia W, Wang F, Zhou Y, Liu Z, Zhan Q, Liu Y, Yu D, Zhai K, Chang J, Qiao Y, Jin G, Liu Z, Shen Y, Guo C, Fu J, Miao X, Tan W, Shen H, Ke Y, Zeng Y, Wu T, Lin D. 2011. Genome-wide association study identifies three new susceptibility loci for esophageal squamous-cell carcinoma in Chinese populations. Nature genetics 43:679-684. DOI: $10.1038 /$ ng. 849 .

Yang B, Luo L, Luo W, Zhou Y, Yang C, Xiong T, Li X, Meng X, Li L, Zhang X, Wang Z, Wang Z. 2017. The genomic dynamics during progression of lung adenocarcinomas. Journal of human genetics 62:783-788. DOI: 10.1038/jhg.2017.40.

Ying J, Shan L, Li J, Zhong L, Xue L, Zhao H, Li L, Langford C, Guo L, Qiu T, Lu N, Tao Q. 2012. Genome-wide screening for genetic alterations in esophageal cancer by aCGH identifies 11q13 amplification oncogenes associated with nodal metastasis. PloS one 7:e39797. DOI: 10.1371/journal.pone.0039797.

Yingsong Lin, Yukari Totsuka, Yutong He, Shogo Kikuchi, Youlin Qiao, Junko Ueda, Wenqiang Wei, Manami Inoue HT. 2013. Epidemiology of Esophageal Cancer in Japan and China. Journal of Epidemiology 23:233-242. DOI: 10.2188/jea.je20120162.

Yokota T, Serizawa M, Hosokawa A, Kusafuka K, Mori K, Sugiyama T, Tsubosa Y, Koh Y. 2018. PIK3CA mutation is a favorable prognostic factor in esophageal cancer: molecular profile by next-generation sequencing using surgically resected formalin-fixed, paraffin- 
embedded tissue. BMC cancer 18:826. DOI: 10.1186/s12885-018-4733-7.

724

Yoshida K, Suetsugu T, Imai T, Matsuhashi N, Yamaguchi K. 2018. Recent advancements in 725 esophageal cancer treatment in Japan. :253-265. DOI: 10.1002/ags3.12174.

726 Zhang X, Kang C, Li N, Liu X, Zhang J, Gao F, Dai L. 2019. Identification of special key genes 727 for alcohol-related hepatocellular carcinoma through bioinformatic analysis. PeerJ $728 \quad 7: e 6375$. DOI: $10.7717 /$ peerj.6375.

729 Zhang L, Zhou Y, Cheng C, Cui H, Cheng L, Kong P, Wang J, Li Y, Chen W, Song B, Wang F, 730 Jia Z, Li L, Li Y, Yang B, Liu J, Shi R, Bi Y, Zhang Y, Wang J, Zhao Z, Hu X, Yang J, Li 731 H, Gao Z, Chen G, Huang X, Yang X, Wan S, Chen C, Li B, Tan Y, Chen L, He M, Xie S, 732 Li X, Zhuang X, Wang M, Xia Z, Luo L, Guo J, Chen X, Zhang Y, Li Q, Liu L, Li Y, 733 Zhang X. 2015. Genomic Analyses Reveal Mutational Signatures and Frequently Altered 734 Genes in Esophageal Squamous Cell Carcinoma. The American Journal of Human Genetics 735 96:597-611. DOI: 10.1016/j.ajhg.2015.02.017.

736

737 738

739

740

741

742

743

744

745

746

Zhen DB, Rabe KG, Gallinger S, Syngal S, Schwartz AG, Goggins MG, Hruban RH, Cote ML, McWilliams RR, Roberts NJ, Cannon-Albright LA, Li D, Moyes K, Wenstrup RJ, Hartman A-R, Seminara D, Klein AP, Petersen GM. 2015. BRCA1, BRCA2, PALB2, and CDKN2A mutations in familial pancreatic cancer: a PACGENE study. Genetics in medicine : official journal of the American College of Medical Genetics 17:569-577. DOI: 10.1038/gim.2014.153.

\section{FIGURE LEGENDS}


747 Figure 1. The landscape of somatic alternations in ESCCs from 20 Japanese patients. Figure

748 1(A) shows potential driver mutations by SNVs/INDELs across the 20 ESCC patients with

749 different mutation types which are coded by different color. Two genes marked with asterisk are

750 significantly mutated genes $(q<0.05)$ detected by the $\mathrm{dNdScv}$ method. Other 12 genes are those

751 recurrently mutated in previous ESCC studies. Top panel shows the number of mutations in all

752 the 20 ESCC cases. Right panel indicates the number and type of mutations for each mutated

753 gene. Mutation types are labelled on the right legend. The middle panel (B) represents the

754 recurrent copy number amplified and deleted regions with the important cancer-related genes

755 detected in ESCC patients. The legend on the right side shows frequency across the ESCC

756 patients. The bottom panel (C) shows the clinical features such as gender, smoking, alcohol

757 drinking status, age and the tumor stages of the ESCC patients.

758

759 Figure 2. Mutational signatures of 20 whole genome of ESCC. (A-F) Characterization of six 760 mutational signatures identified across the ESCC genomes. Patterns of substitutions for each

761 signatures W1-W6 in ESCC. The mutational signatures are presented according to the 96

762 substitution classifications defined by the substitution class and sequence context immediately 3'

763 and 5' to the mutated base. We used SignatureAnalyzer method to determine the six distinct

764 mutational signatures (SNVs) out of the 20 ESCC samples. (A) Signature W1. (B) Signature W2.

765 (C) Signature W3. (D) Signature W4. (E) Signature W5. (F) Signature W6. (G) Mutation burden

766 and contribution of the six mutational signatures (W1-W6) across the ESCC genomes. (H)

767 Boxplot showing the association of mutational signatures and smoking habit of patients with

768 ESCC. Mutational signature W4 displays significant association ( $p$-value $=0.01474)$ with 
769 smoking status of ESCC patients. P-value was calculated using Wilcoxon rank sum test. ns: $P>$ $770 \quad 0.05$

771

772 Figure 3. Somatic structural variations in ESCC. (A-L) Circos plots of SVs and somatic CNAs 773 in 20 ESCC genome. The inner ring represents the SVs: red for intrachromosomal

774 rearrangements, and green for interchromosomal rearrangements. The second ring next to SVs 775 displays the CNAs: red for amplifications and blue for deletions. The outer ring shows the 776 chromosome ideogram. (A-F) ESCCs with 100 or more SVs. (G-L) ESCCs with <100 SVs.

777 (M) Different categories of SVs for instance inversion, translocation, deletion and tandem 778 duplication, and their frequencies in the 20 ESCC genomes. (N) Top genes recurrently affected 779 by SVs across the ESCC samples. Genes that were affected in two or more patients are 780 presented.

781

782

Figure 4. Somatic copy number alterations in specific regions detected by GISTIC 2.0 in ESCC.

783 Significantly observed regions of recurrent amplifications (A) and deletions (B) across samples 784 are shown. Numbers in the left bar in both $(\mathbf{A})$ and $(\mathbf{B})$ refer to the chromosome number. GISTIC 785 scores are presented on top and, q-values (x-axis) indicating the false discovery rate at each locus 786 are shown on a $\log$ scale in both (A) and (B). (C) Common deletions are shown at 9p21.3 region 787 across the ESCC samples by Integrative Genomic Viewer (IGV).

788

789 Figure 5. Venn diagram representing the druggable genes altered by different mutation events

790 in ESCC. The figure shows the common druggable genes, genes having interaction with at least 791 one drug target. These genes were affected by SNVs, SVs and CNAs in ESCC. 
794 Table 1. Summary of the clinical information of the ESCC samples used in this study.

795

796 Total number of figures and tables: 5 figures and 1 table

797 Supplementary information: 6 figures and 11 tables

798 


\section{Figure 1}

The landscape of somatic alternations in ESCCs from 20 Japanese patients.

Figure $1(\mathbf{A})$ shows potential driver mutations by SNVs/INDELs across the 20 ESCC patients with different mutation types which are coded by different color. Two genes marked with asterisk are significantly mutated genes $(q<0.05)$ detected by the dNdScv method. Other 12 genes are those recurrently mutated in previous ESCC studies. Top panel shows the number of mutations in all the $20 \mathrm{ESCC}$ cases. Right panel indicates the number and type of mutations for each mutated gene. Mutation types are labelled on the right legend. The middle panel (B) represents the recurrent copy number amplified and deleted regions with the important cancer-related genes detected in ESCC patients. The legend on the right side shows frequency across the ESCC patients. The bottom panel (C) shows the clinical features such as gender, smoking, alcohol drinking status, age and the tumor stages of the ESCC patients. 


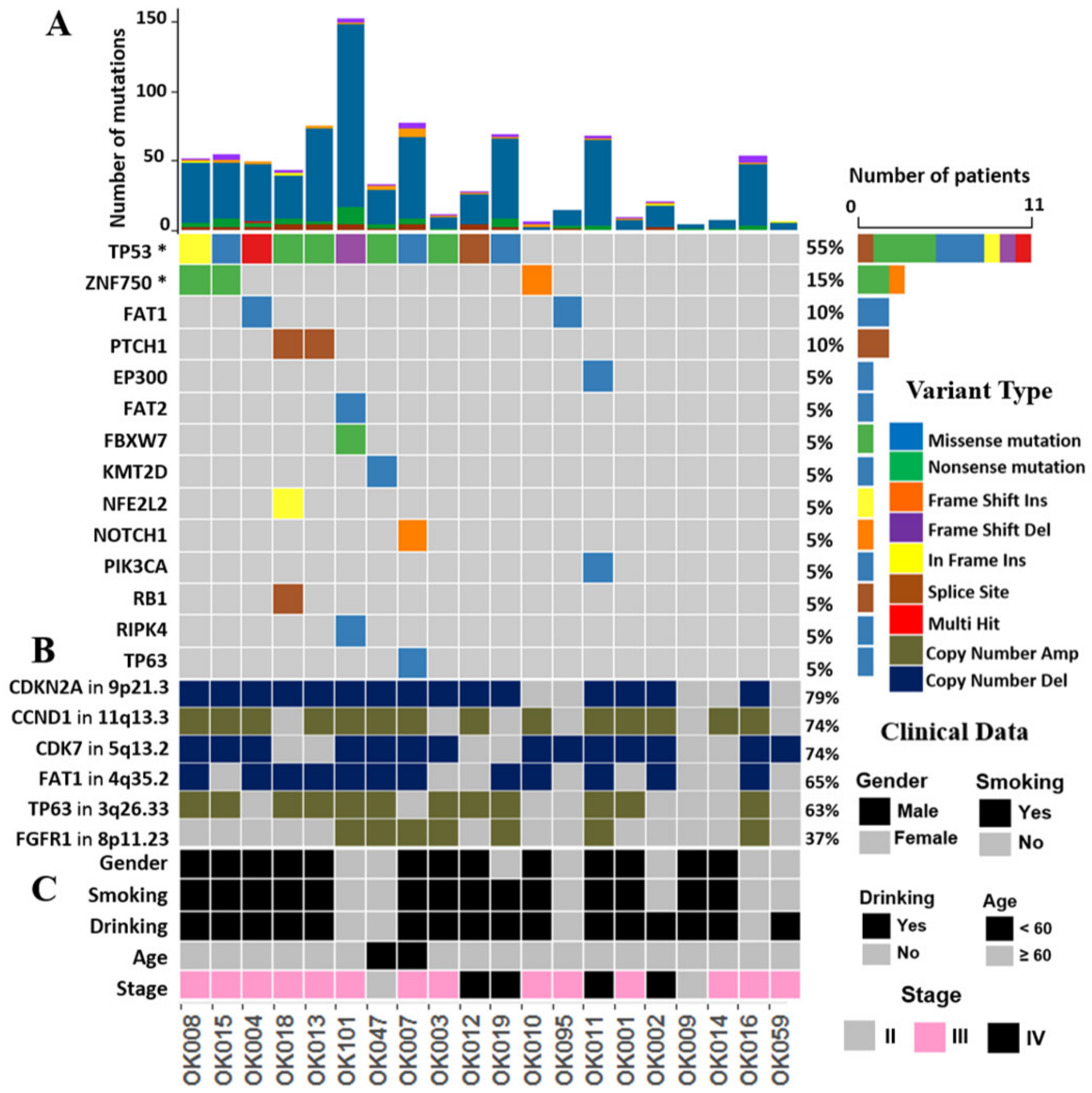




\section{Figure 2}

Mutational signatures of 20 whole genome of ESCC.

(A-F) Characterization of six mutational signatures identified across the ESCC genomes.

Patterns of substitutions for each signatures W1-W6 in ESCC. The mutational signatures are presented according to the 96 substitution classifications defined by the substitution class and sequence context immediately $3^{\prime}$ and $5^{\prime}$ to the mutated base. We used

SignatureAnalyzer method to determine the six distinct mutational signatures (SNVs) out of the 20 ESCC samples. (A) Signature W1. (B) Signature W2. (C) Signature W3. (D) Signature W4. (E) Signature W5. (F) Signature W6. (G) Mutation burden and contribution of the six mutational signatures (W1-W6) across the ESCC genomes. (H) Boxplot showing the association of mutational signatures and smoking habit of patients with ESCC. Mutational signature W4 displays significant association ( $p$-value $=0.01474$ ) with smoking status of ESCC patients. P-value was calculated using Wilcoxon rank sum test. ns: $P>0.05$. 

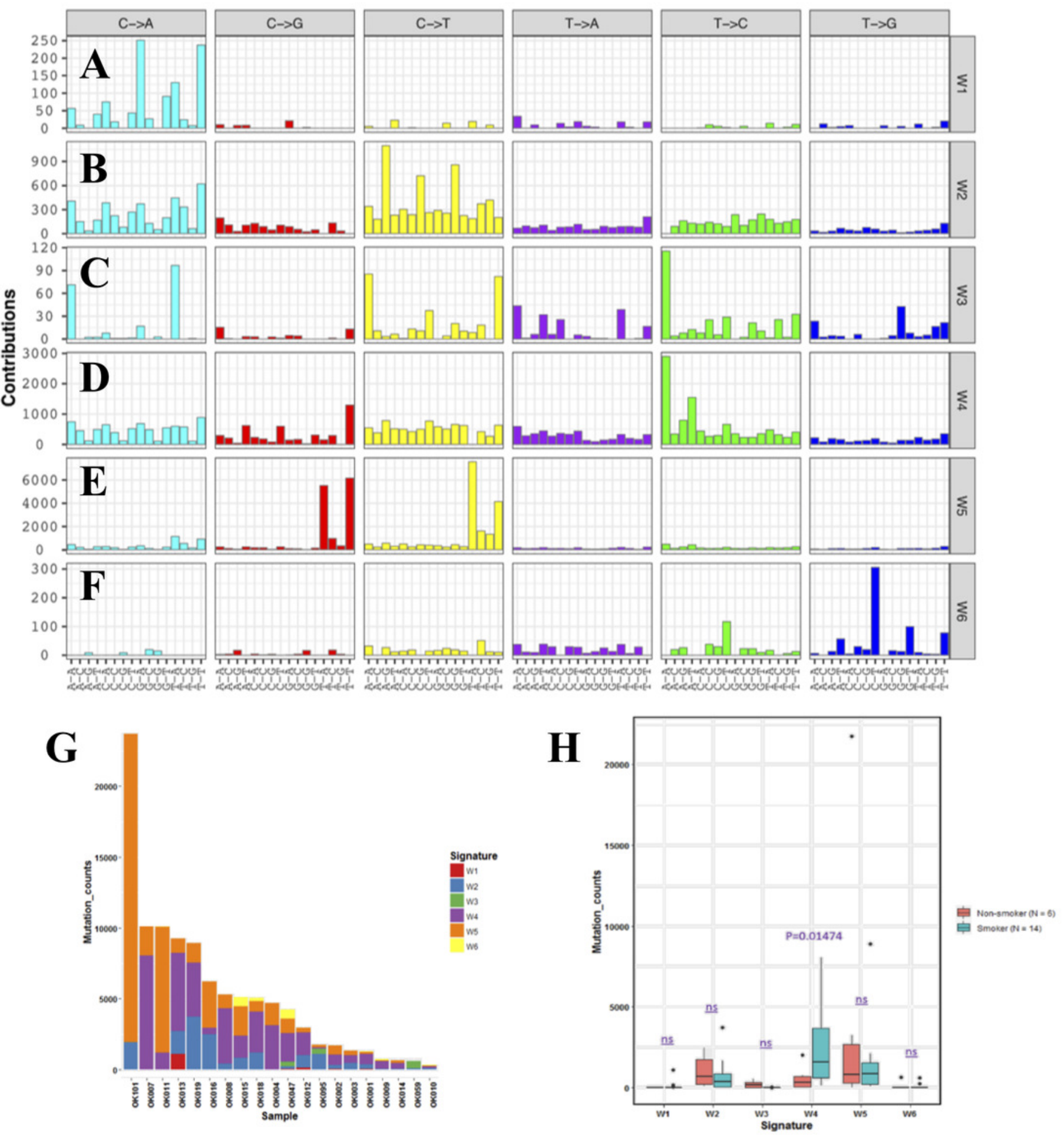


\section{Figure 3}

Somatic structural variations in ESCC.

(A-L) Circos plots of SVs and somatic CNAs in 20 ESCC genome. The inner ring represents the SVs: red for intrachromosomal rearrangements, and green for interchromosomal rearrangements. The second ring next to SVs displays the CNAs: red for amplifications and blue for deletions. The outer ring shows the chromosome ideogram. (A-F) ESCCs with 100 or more SVs. (G-L) ESCCs with <100 SVs. (M) Different categories of SVs for instance inversion, translocation, deletion and tandem duplication, and their frequencies in the $20 \mathrm{ESCC}$ genomes. (N) Top genes recurrently affected by SVs across the ESCC samples. Genes that were affected in two or more patients are presented. 
A

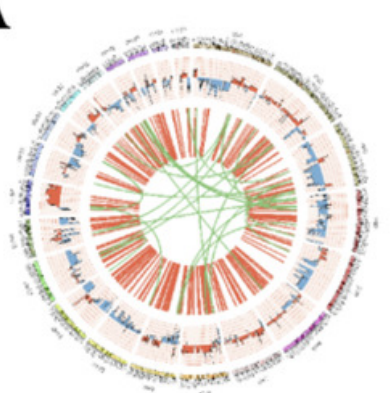

OK007

B

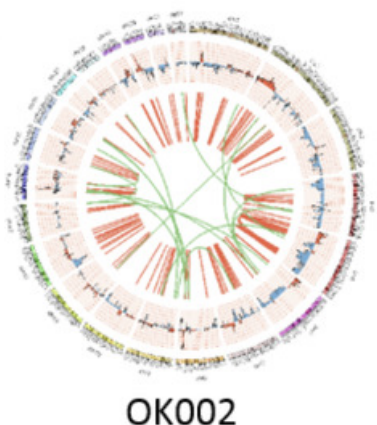

C

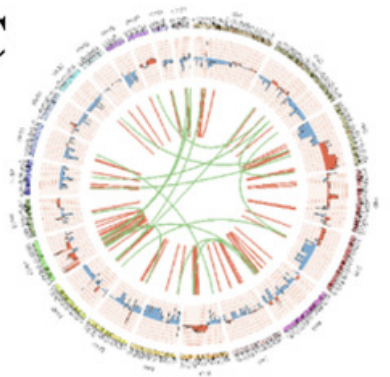

$\uparrow \quad$ OK015

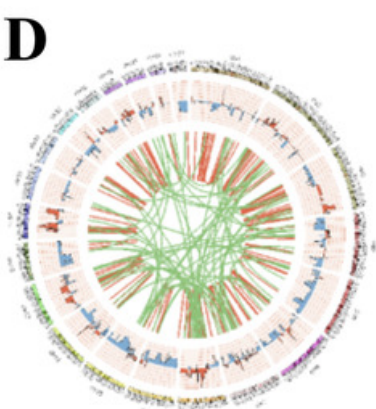

OK008

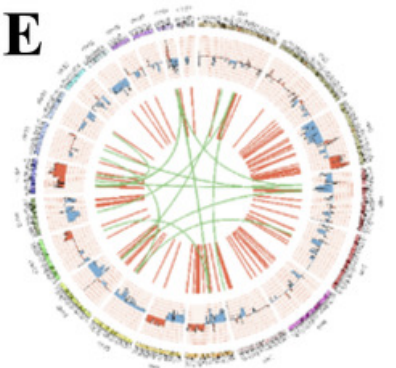

OK013

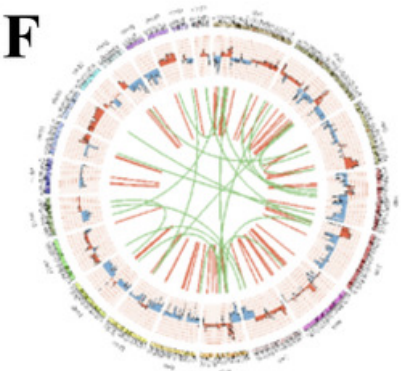

OK016

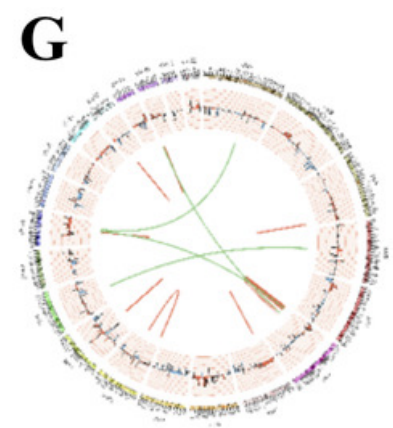

OK010

H

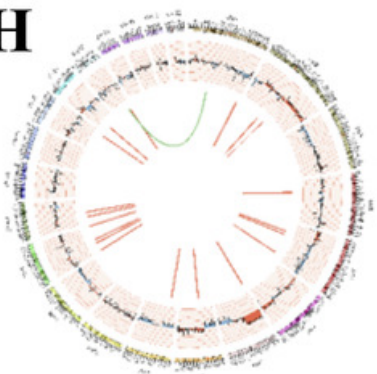

OK009

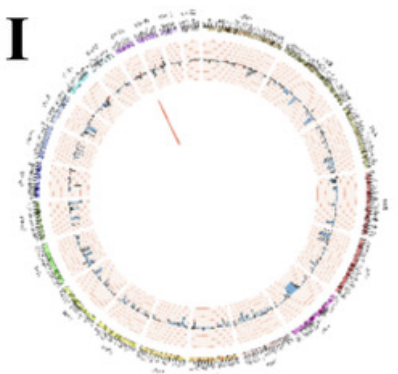

OK059

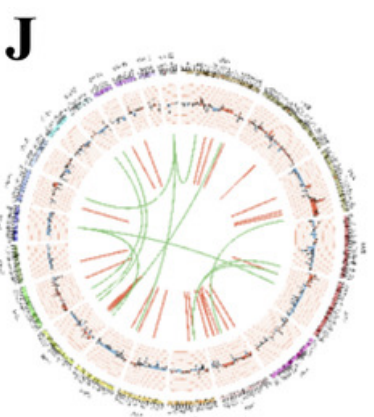

OK003

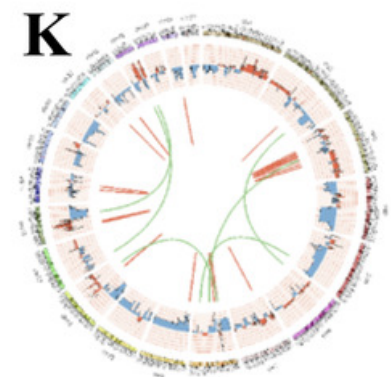

OK101

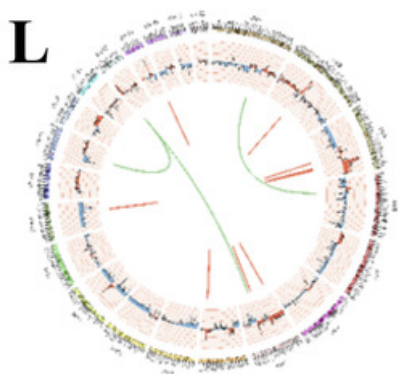

OK047

No. of SVs $\geq 100$

No. of SVs $<100$

Interchromosomal interaction Intrachromosomal interaction

Copy number deletion

Copy number amplification

M

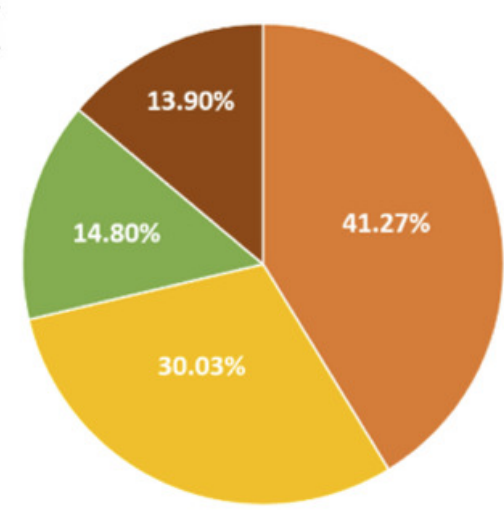

Inversion Translocation

Deletion

Tandem Duplicatio

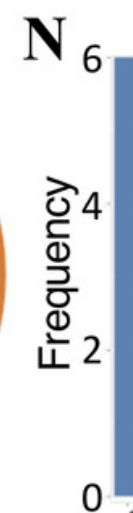

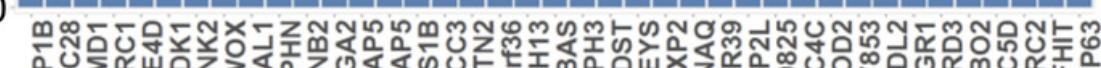

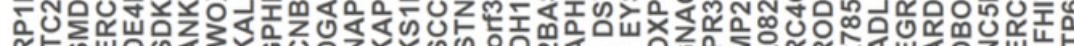

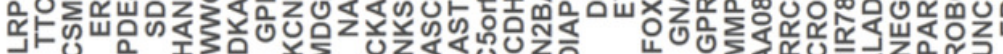




\section{Figure 4}

Somatic copy number alterations in specific regions detected by GISTIC 2.0 in ESCC.

Significantly observed regions of recurrent amplifications (A) and deletions (B) across

samples are shown. Numbers in the left bar in both (A) and (B) refer to the chromosome number. GISTIC scores are presented on top and, $q$-values ( $x$-axis) indicating the false discovery rate at each locus are shown on a log scale in both (A) and (B). (C) Common deletions are shown at 9p21.3 region across the ESCC samples by Integrative Genomic Viewer (IGV). 

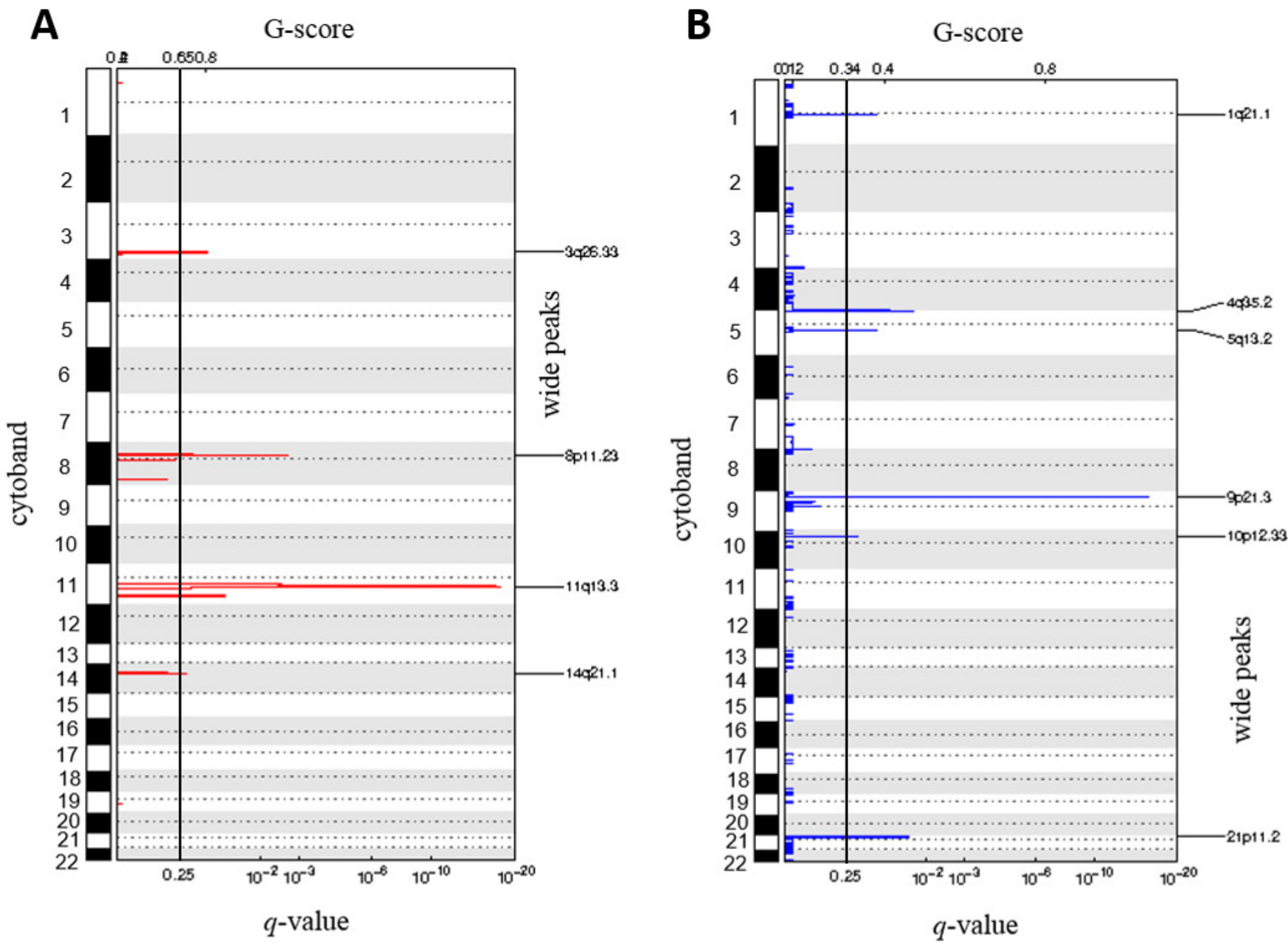

C

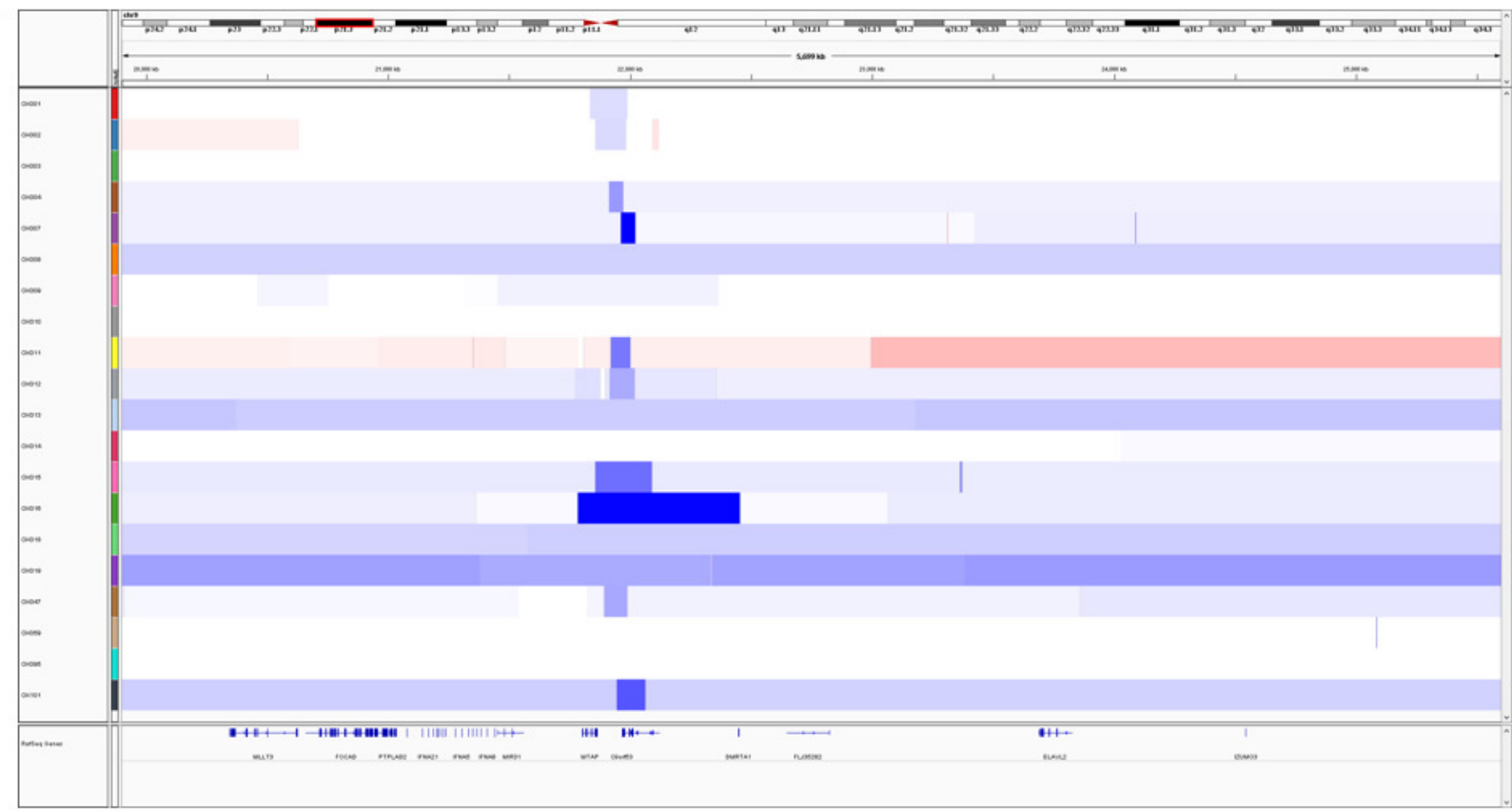


Figure 5

Venn diagram representing the druggable genes altered by different mutation events in ESCC.

The figure shows the common druggable genes, genes having interaction with at least one drug target. These genes were affected by SNVs, SVs and CNAs in ESCC. 


\section{SNVs}

TP53, NOTCH1, RB1, JAK2, ACAN, LAMA3, EPHA5, HPSE, PDE7A, GRM8, COL4A3, LRP2, SCN9A, RICTOR, EPHA7, ITPR3

189

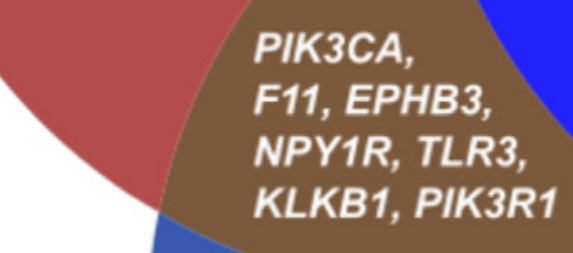

PIK3CA, F11, EPHB3, NPY1R, TLR3, KLKB1, PIK3R1

\section{0}

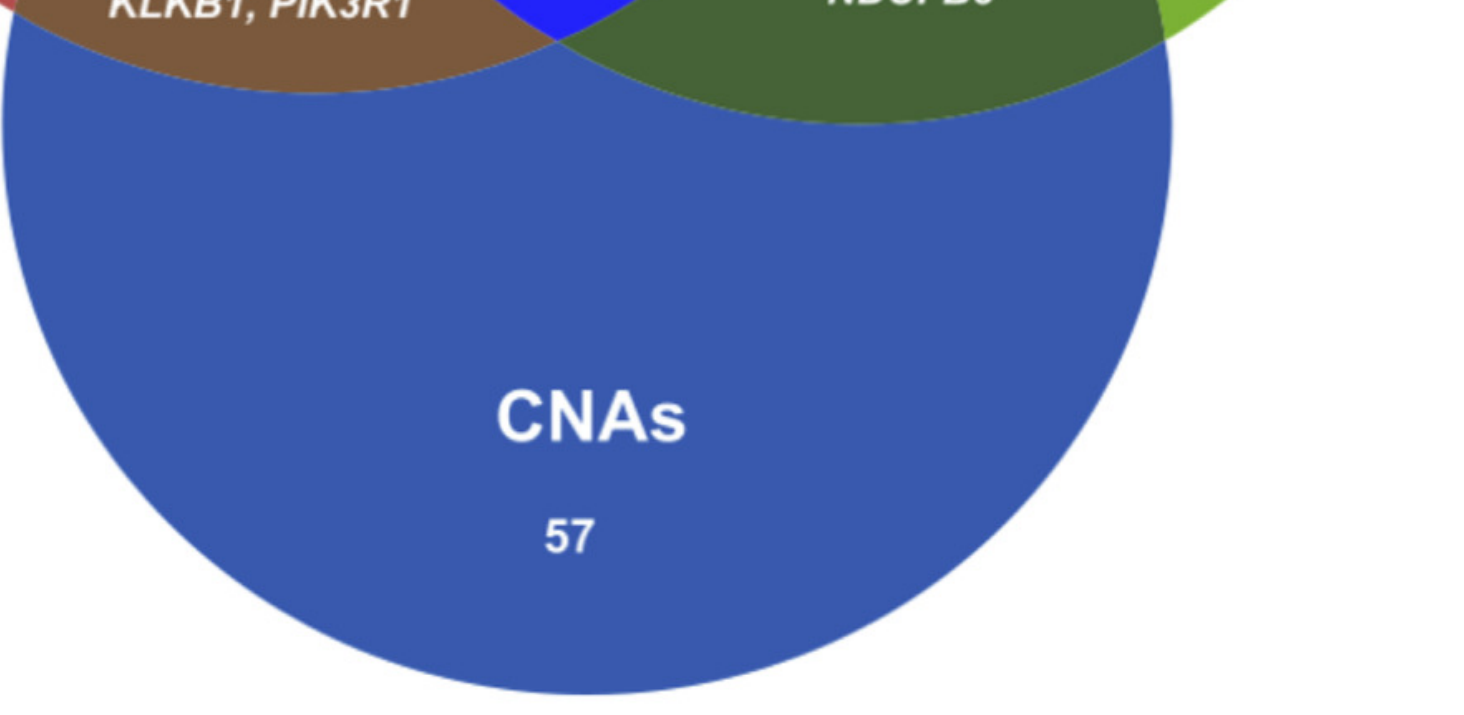




\section{Table 1 (on next page)}

Summary of the clinical information of the ESCC samples used in this study 
1 Table 1: Summary of the clinical information of the ESCC samples used in this study.

\begin{tabular}{|c|c|c|}
\hline \multirow[t]{2}{*}{ Sex } & Male & 13 \\
\hline & Female & 7 \\
\hline \multirow[t]{2}{*}{ Histology } & Squamous cell carcinoma & 19 \\
\hline & Basaloid squamous cell carcinoma & 1 \\
\hline \multirow[t]{5}{*}{ Tumor location } & Cervical esophagus & 1 \\
\hline & Upper thoracic esophagus & 2 \\
\hline & Middle thoracic esophagus & 13 \\
\hline & Lower thoracic esophagus & 3 \\
\hline & Abdominal esophagus & 1 \\
\hline \multirow[t]{3}{*}{ Tumor stage } & II & 2 \\
\hline & III & 14 \\
\hline & IV & 4 \\
\hline \multirow[t]{2}{*}{ Age } & $\geq 60$ & 18 \\
\hline & $<60$ & 2 \\
\hline \multirow[t]{2}{*}{ Smoking status } & Smoker & 14 \\
\hline & Non-smoker & 6 \\
\hline \multirow[t]{2}{*}{ Alcohol drinking status } & Drinker & 16 \\
\hline & Non-drinker & 4 \\
\hline \multirow[t]{2}{*}{ Response to chemotherapy } & Responder & 10 \\
\hline & Non-responder & 10 \\
\hline
\end{tabular}

2

3

4

5

6 
